\title{
Near-infrared photometry of carbon stars in the Sagittarius dwarf irregular galaxy and DDO 210
}

\author{
M. Gullieuszik ${ }^{1,2}$, M. Rejkuba ${ }^{3}$, M. R. Cioni ${ }^{4}$, H. J. Habing ${ }^{5}$, and E. V. Held ${ }^{1}$ \\ 1 Osservatorio Astronomico di Padova, INAF, vicolo dell'Osservatorio 5, 35122 Padova, Italy \\ e-mail: [marco.gullieuszik; enrico.held]@oapd.inaf.it \\ 2 Dipartimento di Astronomia, Università di Padova vicolo dell'Osservatorio 2, 35122 Padova, Italy \\ 3 European Southern Observatory, Karl-Schwarzschild-Strasse 2, 85748 Garching, Germany \\ e-mail: mrejkuba@eso.org \\ ${ }^{4}$ SUPA, School of Physics, University of Edinburgh, IfA, Blackford Hill, Edinburgh EH9 3HJ, UK \\ e-mail: mrc@roe.ac.uk \\ 5 Sterrewacht Leiden, Niels Bohrweg 2, 2333 RA Leiden, The Netherlands \\ e-mail: habing@strw.leidenuniv.nl
}

Received 30 November 2006 / Accepted 22 May 2007

ABSTRACT

\begin{abstract}
Aims. We investigate the intermediate-age asymptotic giant branch stellar population of two Local Group dwarf irregular galaxies to characterize their carbon star population in near-infrared (IR).

Methods. Our work is based on near-IR photometry complemented with optical ground based and Hubble Space Telescope (HST) photometry. Near-IR photometry is based on our and archival $J$ and $K_{\mathrm{s}}$-band images from SOFI near-IR array of the ESO New Technology Telescope (NTT). Optical photometry for DDO 210 is from the EMMI optical imager of ESO NTT, while the SagDIG optical data come from Momany et al. (2005, A\&A, 439, 111).

Results. We show that near-IR photometry is a very powerful tool for carbon star detection. We recovered two out of three previouslyknown carbon stars in DDO 210 and discovered six additional objects in this galaxy which have optical and near-IR colors consistent with carbon giants. This brings the total number of bona fide C-star candidates in DDO 210 to nine. However, to confirm the nature of these objects additional higher spatial resolution imaging or spectroscopic data are necessary.

We detected a large population of C-star candidates in SagDIG, 18 of which were previously identified in Demers \& Battinelli (2002, AJ, 123, 238) and Cook (1987, Ph.D. Thesis), and six new bona fide carbon stars. We present their optical and near-IR colors and use their luminosity function to put constraints on the star formation history (SFH) in this dwarf irregular galaxy.
\end{abstract}

Key words. galaxies: individual: SagDIG - galaxies: individual: DDO 210 - galaxies: stellar content - stars: AGB and post-AGB stars: carbon

\section{Introduction}

The Asymptotic Giant Branch (AGB) is an important, although short-lived phase during the final evolutionary stages of low and intermediate mass stars. In unresolved galaxies with intermediate-age stellar populations AGB stars provide a major contribution to the integrated light (Renzini \& Buzzoni 1986). A good description of this evolutionary phase is therefore fundamental to understand the integrated properties of high redshift galaxies (e.g. Maraston et al. 2006). Its importance for the chemical evolution and SFH in external galaxies is shaded by the fact that this is also one of the least known evolutionary phases. Large samples of AGB stars in systems with well defined distances, metallicities and SFHs are necessary to constrain the theoretical models which predict their properties. In addition, Battinelli \& Demers (2005) considered the possible standard candle aspect of AGB carbon stars and concluded that the mean I-band magnitude of $\mathrm{C}$-stars can provide reliable distance

^ Based on data collected at the European Southern Observatory, La Silla, Chile, Proposal No. 71.D-0560, and archived ESO data from the Proposal No. 61.E-0273.

$\star \star$ Appendix $\mathrm{A}$ is only available in electronic form at http://www. aanda.org determinations up to $\sim 2 \mathrm{Mpc}$ with currently available groundbased telescopes.

A systematic census of AGB C-stars in Local Group (LG) galaxies was made over the last few years using the four-filter technique with two optical broad-band and two narrow-band filters (Albert et al. 2000; Battinelli \& Demers 2000; Nowotny et al. 2001).

A different approach (e.g. Cioni \& Habing 2005; Gullieuszik et al. 2005; Kang et al. 2005) is based on the combination of one optical filter with $J$ and $K_{\mathrm{s}}$ near-IR broad-band filters. In both near-IR broad-band and in optical broad plus narrow-band filter techniques, C and M-type stars can be easily separated in colorcolor diagrams. However the selection criteria are somewhat different, and both methods are likely to miss some C-stars, or to select some that would appear as M-type stars when considering the alternative method (e.g. Demers et al. 2006, and later in this work). More specifically the advantages of near-IR over purely optical techniques are: (i) extinction in the near-IR is much lower than in the optical, reducing the problems with differential reddening that can be present in star forming dwarf irregular (dIrr) galaxies; (ii) the spectral energy distribution (SED) of AGB stars peaks in the near-IR and makes them easily distinguishable from red giant branch (RGB) stars; (iii) from $J-K_{\mathrm{S}}$ color and 
Table 1. Main parameters of the observed galaxies.

\begin{tabular}{ccr}
\hline \hline & SagDIG & \multicolumn{1}{c}{ DDO 210 } \\
\hline$(m-M)_{0}$ & $25.10^{(1)}$ & $24.89^{(2)}$ \\
$E_{(B-V)}$ & $0.12^{(1)}$ & $0.03^{(2)}$ \\
{$[\mathrm{Fe} / \mathrm{H}]$} & $-2.0^{(1)}$ & $-1.9^{(2)},-1.3^{(3)}$ \\
$M_{v}$ & -12.3 & $-10.9^{(2)}$ \\
$M_{\mathrm{HI}} / 10^{6} M_{\odot}$ & $8^{(4)}$ & $3^{(4)}$ \\
\hline
\end{tabular}

(1) Momany et al. (2005); (2) Lee et al. (1999); (3) McConnachie et al. (2006); ${ }^{(4)}$ Lo et al. (1993).

$K_{\mathrm{s}}$ magnitude it is possible to derive precise bolometric magnitudes; and (iv) with the development of adaptive optics at large telescopes (NACO at VLT, adaptive optics at the next generation of Extremely Large Telescopes), operating principally in the near-IR, it is important to know well the observational characteristics and have well prepared models for the interpretation of the data of more distant objects that will shortly be observed. It is important to know how these bright near-IR sources, the first to be observed in a distant galaxy, behave with age and metallicity.

Following this approach we present the first $J$ and $K_{\mathrm{s}}$ photometry of SagDIG and DDO 210, two southern Local Group metal-poor dIrr galaxies. Near-IR data are complemented with ground based $I$-band photometry for DDO 210, and $V$, I ACS/HST photometry by Momany et al. (2005) for SagDIG.

DDO 210, also known as Aquarius dIrr, is one of the faintest dwarf galaxies (Lee et al. 1999), while SagDIG is one of the brightest (see Table 1). The number of C-stars is roughly a function of the galaxy mass or absolute magnitude (Groenewegen 2004; Battinelli \& Demers 2005), so the number of expected $\mathrm{C}$-stars in the two galaxies is very different. As a part of a homogeneous survey, Battinelli \& Demers (2000) found only three C-stars in DDO 210, and Demers \& Battinelli (2002) found 16 in SagDIG.

The aim of this paper is to investigate the AGB stars of SagDIG and DDO 210 and in particular to characterize the near-IR properties of their C-star population.

\section{Observations}

\subsection{SagDIG}

Observations were made during August 2003 with the SOFI (Moorwood et al. 1998) camera mounted on the NTT telescope at ESO/La Silla Observatory. The camera is equipped with a $1024 \times 1024$ Hawaii CCD. In Large Field mode, with a spatial resolution of $0.288 \mathrm{pix}^{-1}$, the field of view is $4.9 \times 4.9$. Our data set consists of $24 J$ frames and $48 K_{\text {s }}$ frames centered on SagDIG (see Table 2 for a log of the observations). SagDIG is close in projection to the Galactic center, so the number of contaminating foreground stars expected in the color magnitude diagram (CMD) is high. To have an estimate of the contribution of the foreground stellar population we also observed an external field, located beyond the tidal radius of SagDIG.

$J$ and $K_{\mathrm{s}}$ data from the ESO archive (prog ID. 61.E-0273) were also used. Observations were carried out with SOFI in Large Field mode during two nights in August 1998 and the data set consists of $18 \mathrm{~J}$ frames and $108 K_{\mathrm{s}}$ frame centered on SagDIG.
Table 2. Observing Log.

\begin{tabular}{|c|c|c|c|c|c|}
\hline \multicolumn{2}{|r|}{ Night } & Filter & $N_{\text {TOT }}$ & $N_{\text {Used }}$ & DIT $(s) \times$ NDIT \\
\hline \multicolumn{6}{|c|}{ SagDIG } \\
\hline \multicolumn{6}{|c|}{$\alpha(\mathrm{J} 2000)=19^{\mathrm{h}} 29^{\mathrm{m}} 59^{\mathrm{s}}, \delta(\mathrm{J} 2000)=-17^{\circ} 40^{\prime} 42^{\prime \prime}$} \\
\hline $11-12$ & 2 Aug. 1998 & $J$ & 18 & 9 & $20.0 \times 6$ \\
\hline $11-12$ & Aug. 1998 & $K_{\mathrm{s}}$ & 108 & 108 & $10.0 \times 6$ \\
\hline $1-2$ & Aug. 2003 & $J$ & 24 & 13 & $10.0 \times 6$ \\
\hline $1-2$ & Aug. 2003 & $K_{\mathrm{s}}$ & 48 & 31 & $6.0 \times 10$ \\
\hline \multicolumn{6}{|c|}{ SagDIG control field } \\
\hline \multicolumn{6}{|c|}{$\alpha(\mathrm{J} 2000)=19^{\mathrm{h}} 29^{\mathrm{m}} 24^{\mathrm{s}}, \delta(\mathrm{J} 2000)=-17^{\circ} 32^{\prime} 22^{\prime \prime}$} \\
\hline $1-2$ & Aug. 2003 & $J$ & 24 & 24 & $10.0 \times 6$ \\
\hline $1-2$ & Aug. 2003 & $K_{\mathrm{s}}$ & 48 & 48 & $6.0 \times 10$ \\
\hline \multicolumn{6}{|c|}{ DDO 210} \\
\hline \multicolumn{6}{|c|}{$\alpha(\mathrm{J} 2000)=20^{\mathrm{h}} 47^{\mathrm{m}} 01^{\mathrm{s}}, \delta(\mathrm{J} 2000)=-12^{\circ} 51^{\prime} 00^{\prime \prime}$} \\
\hline $1-2$ & Aug. 2003 & $J$ & 24 & 24 & $10.0 \times 6$ \\
\hline $1-2$ & Aug. 2003 & $K_{\mathrm{s}}$ & 48 & 48 & $6.0 \times 10$ \\
\hline 1 & Aug. 2003 & $I$ & 4 & 4 & $300.0 \times 1$ \\
\hline 1 & Aug. 2003 & $I$ & 2 & 2 & $10.0 \times 1$ \\
\hline
\end{tabular}

\section{2. $D D O 210$}

Two contiguous fields in DDO 210 were observed with SOFI, following the same strategy adopted for SagDIG. In addition, four deep and two shallow images in the $I$-band were taken with EMMI, the NTT multi-mode instrument (Dekker et al. 1986), in RILD mode. The camera is equipped with two $2048 \times 4096$ detectors. In $2 \times 2$ binning mode the pixel scale is 0. o $^{\prime} 33$ pix $^{-1}$ providing a field of view of $9.9 \times 9$ '.9, so with one pointing we could cover both SOFI fields. Observations are summarized in Table 2.

\section{Data reduction}

\subsection{SOFI data}

Following the SOFI User's manual we did not apply dark frame correction, because the dark current is low and "for broad-band imaging the dark frames is a poor approximation of the underlying bias pattern, so subtracting it serves no real purpose”.

Special Flats frames were obtained multiplying the flat field frames with the so-called illumination correction frames that were downloaded from the SOFI webpages ${ }^{1}$.

Reduction of the data was done using standard IRAF scripts, independently per night and filter, following the recipes described by Rejkuba et al. (2001).

For each image, the sky subtraction was made using the five frames closest in time as "sky" frames and creating object masks with SEXTRACTOR to avoid overestimation of the sky level. The final sky frame was computed averaging all five "sky" frames with the objects masked. The corresponding final sky frame was subtracted from each image which was then divided by the Special Flat frame. Finally the mean value of the sky frame was added.

Given the relatively large number of SagDIG frames, only those with good seeing (better than 1 .'1) were used. The resulting number of frames that are used below is given in Col. 4 of Table 2. For DDO 210, we used all available frames.

Aperture photometry was done on each image using DAOPHOT (Stetson 1987) and the relative shifts between all frames belonging to a single epoch (1998 and 2003) were calculated with DAOMASTER (Stetson 1993). The frames were finally registered with imalign and combined with imcombine

\footnotetext{
1 http://www.ls.eso.org/lasilla/sciops/ntt/sofi/ images/fits/Archive/
} 
tasks in IRAF. The final result are the master $J$ and $K_{\mathrm{s}}$ images of each epoch.

A posteriori we verified that rejecting the frames with poorer seeing leads to deeper master images with a higher $\mathrm{S} / \mathrm{N}$ ratio.

\subsection{EMMI data}

The frames delivered by the EMMI CCD are in the Multi Extension Fits Format. To read them and re-create a single image, a MIDAS script, provided by the La Silla Observatory, was used ${ }^{2}$. Images were then processed within IRAF using the $x c c d r e d$ package. The reduction steps included bias subtraction and division by normalized twilight flat-field images.

\section{Photometry}

\subsection{SOFI data}

Point-spread-function (PSF) photometry was performed using DAOPHOT/ALLFRAME (Stetson 1987, 1994) on each single epoch master image. The PSF was generated with a PENNY function with a quadratic dependence on position on the frame. The photometry of the two epochs was finally combined with DAOMASTER. We also produced a catalogue from the external SagDIG field observations, following the same procedure previously described. In order to use the latter for estimating the contribution of the foreground stellar population, we generated a catalogue using only our observations (Aug. 2003 run).

All master images were astrometrically calibrated using the 2MASS point source catalogue (Cutri et al. 2003) as the reference. The relative astrometric accuracy is always better than $0 . ' 3$.

The color term between the LCO photometric system defined by Persson et al. (1998) and that of SOFI is negligible (Gullieuszik et al. 2007). Moreover Carpenter (2001) shows that the LCO and the 2MASS systems are equivalent. Given these, photometric calibration was done applying the zero point difference between our uncalibrated catalogue and the 2MASS point source catalogue (Cutri et al. 2003), as shown in Fig. 1 for SagDIG. Photometric calibration of DDO 210 SOFI data was done in the same way.

\subsection{EMMI data}

PSF fitting photometry was performed, using standard DAOPHOT/ALLFRAME procedures. The PSF was generated with a PENNY function with a quadratic dependence on position on the frame. The list of objects used as input for ALLFRAME was generated on a median image produced from all six EMMI frames. The final ALLFRAME run was performed on all six frames, and the resulting catalogues were generated with DAOMASTER. Instrumental magnitudes have been corrected for aperture corrections. These corrections have been derived for each EMMI chip separately using several bright and isolated stars. The photometry was calibrated using the solution derived by Rejkuba et al. (2005) for the same observing night:

$i=I-25.614+0.054 \times$ Airmass $[+0.040 *(V-I)]$

where $i$ are aperture-corrected instrumental magnitudes and $I$ are magnitudes calibrated to the standard Johnson/Kron-Cousins photometric system. We have no $V$-band observations, so the $(V-I)$ color term was ignored. The resulting calibration of the

\footnotetext{
${ }^{2}$ http://www.ls.eso.org/lasilla/sciops/ntt/emmi/ quickred/inemmi.prg
}
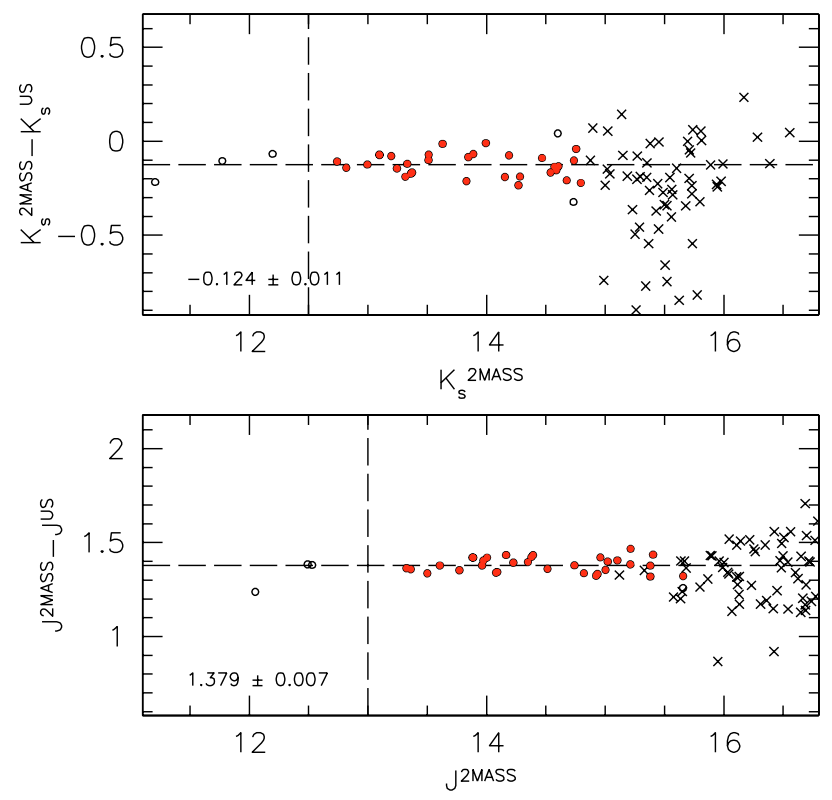

Fig. 1. Zero point differences between our SagDIG uncalibrated magnitudes and the 2MASS point source catalogue. Crosses are 2MASS sources with $S / N<10$ that were not used. Stars marked as open circles were rejected after a $\kappa-\sigma$ clipping. We also rejected bright objects to avoid possible saturation problems.

I photometry is indeed uncertain, but for our purpose this is not a major limitation. The optical data of DD0 210 are used only marginally and our conclusions are not affected by the accuracy of the EMMI $I$-band photometric calibration. However the error in the $I$ magnitude due to the color term should not exceed 0.1 for a single star. Considering also the uncertainties on the aperture correction and on the calibrating relation, we estimated that the final error associated to $I$-band is $0.15 \mathrm{mag}$.

\section{Color-magnitude diagrams}

\subsection{DDO 210}

We have selected stellar objects using the SHARP image quality diagnostic provided by ALLFRAME. Isolated bright stars have a shape very similar to the adopted PSF and a SHARP value close to zero. Unresolved galaxies with elliptical shapes or unrecognized blended stars have SHARP values significantly greater than zero. Cosmic rays and bad pixels have SHARP values below zero. The SHARP value for all objects in our catalogue are plotted in left panel of Fig. 2 as a function of $K_{\mathrm{s}}$ magnitude. The increase of the spread of SHARP values of stellar objects at fainter magnitudes is due to the lower $\mathrm{S} / \mathrm{N}$ ratio at these magnitudes. On the other hand, the size of fainter unresolved galaxies is smaller and some of these could have shapes and sizes similar to the stellar PSF. As a consequence, the sequences of stellar objects and unresolved galaxies/blends merge together at $K_{\mathrm{s}} \sim 19$. The solid line in Fig. 2 shows the function we defined to select stellar objects. At fainter magnitudes, contamination by some unresolved galaxies/blends is possible, but a more severe selection would exclude some faint stars with low $\mathrm{S} / \mathrm{N}$ ratio.

The near-IR $J K_{\mathrm{S}}$ CMD of DDO 210 is shown in Fig. 2. The two almost-vertical sequences at $J-K_{\mathrm{s}} \sim 0.6$ and $J-K_{\mathrm{s}} \sim 0.9$ are populated by foreground Galactic stars (e.g. Nikolaev \& Weinberg 2000). Adopting the parameters reported in Table 1 and the calibration of Valenti et al. (2004) the tip of the RGB 

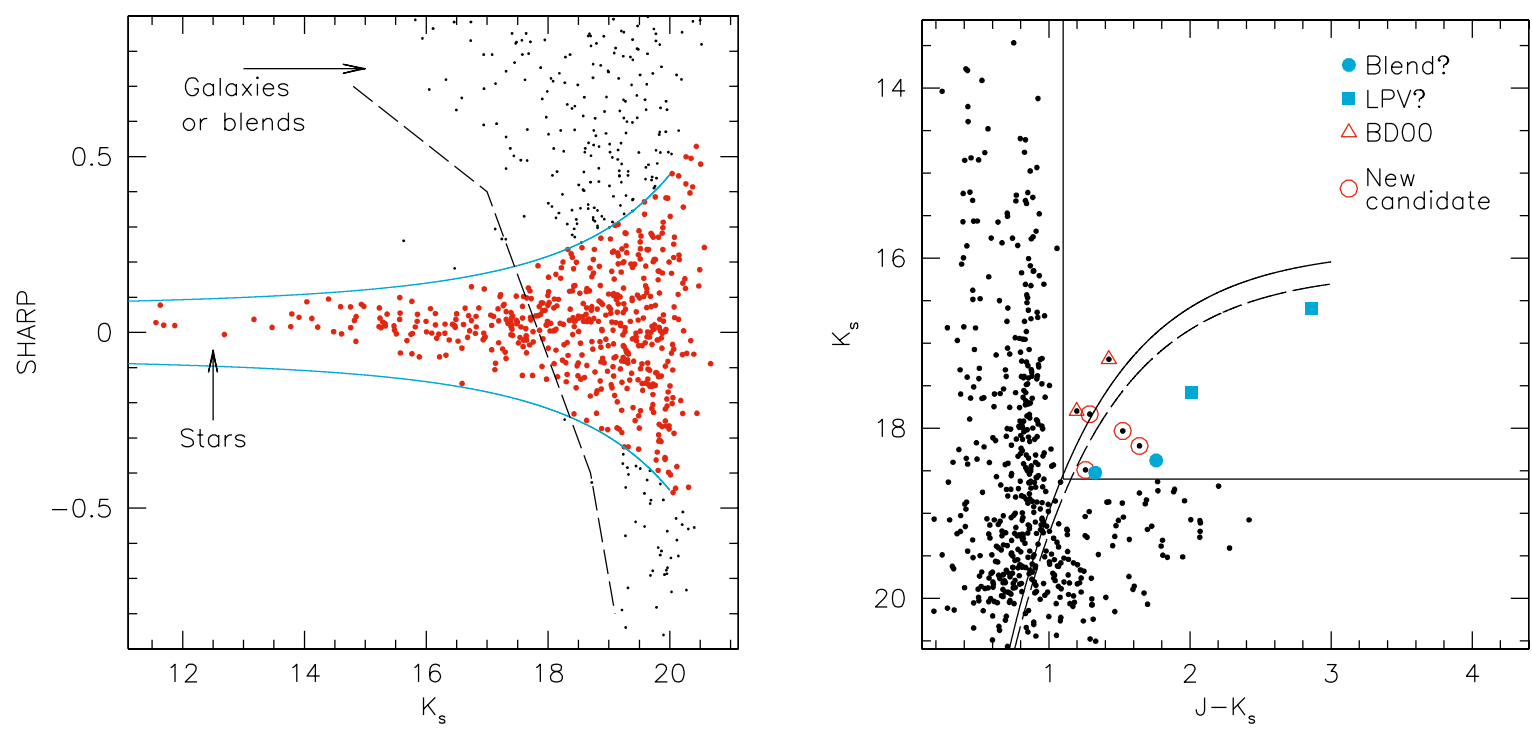

Fig. 2. Left panel: the SHARP parameter as a function of $K_{\mathrm{S}}$ magnitude. Solid lines show the criterion adopted to select stellar objects (larger dots). Dashed lines indicate the limits of the region occupied by unresolved galaxies and photometric blends. Right panel: the near-IR CMD in the field of DDO 210 and the carbon stars identified by Battinelli \& Demers (2000). Probable photometric blends are shown as filled circles, while squares are candidate LPVs. Solid lines show the selection criterion for candidate C-stars. The four new candidates are shown by empty circles. For comparison we also show the mean carbon star color-magnitude relation from Totten et al. (2000) assuming a distance modulus of 24.89 (Lee et al. 1999, solid line) 25.15 (McConnachie et al. 2006, dashed line).

(TRGB) is expected to be found at $J-K_{\mathrm{s}}=0.7$ and $K_{\mathrm{s}}=19.2^{3}$. The latter was calculated assuming $[\mathrm{M} / \mathrm{H}]=[\mathrm{Fe} / \mathrm{H}]([\alpha / \mathrm{Fe}]=0)$ and the Rieke \& Lebofsky (1985) reddening law. The photometric errors and the contamination from foreground stars are very high in the region of the RGB, so it is not visible in the CMD.

In near-IR CMDs oxygen-rich $\mathrm{M}$ giants are found along a sequence that is aligned with the RGB brighter than the RGB tip. Carbon stars are found along a red tail which is clearly separated from M-stars and foreground stars sequences (e.g. Cioni \& Habing 2005; Kang et al. 2005; Gullieuszik et al. 2007). We can therefore conclude that stars found at colors significantly redder than the sequence at $J-K_{\mathrm{s}} \sim 0.9$ in our CMD, are candidate C-stars. The only other objects that could be found in this region are red background galaxies, but these should be found at relatively faint magnitudes. Some AGB stars are surrounded by a dusty circumstellar envelope lost by stellar winds and therefore are found at redder color than normal AGB stars. Indeed, some red stars in our CMD could be dust-enshrouded M-stars. Without additional low resolution spectroscopy they are indistinguishable from C-giants. However, in low metallicity populations such as those present in DDO 210 and also in SagDIG, one expects to find a much higher frequency of $\mathrm{C}$-rich with respect to O-rich AGB stars (e.g. Groenewegen 2004).

Battinelli \& Demers (2000) found three carbon stars in DDO 210. One of them is outside our field of view; the other two are reported in Fig. 2. For comparison, the main locus of C-stars in LG dwarf galaxies obtained by Totten et al. (2000) is also shown. We scaled it to the distance of DDO 210, adopting both the distance derived by Lee et al. (1999) and the higher

3 The calculations were made using the global metallicity $[\mathrm{M} / \mathrm{H}]$, which is related to the $[\mathrm{Fe} / \mathrm{H}]$ iron abundance by taking into account the $\alpha$ elements overabundance $[\alpha / \mathrm{Fe}]$ (Salaris et al. 1993):

$[\mathrm{M} / \mathrm{H}]=[\mathrm{Fe} / \mathrm{H}]+\log \left(0.638 f_{\alpha}+0.362\right) ; \quad f_{\alpha}=10^{[\alpha / \mathrm{Fe}]}$.

The choice of $[\mathrm{M} / \mathrm{H}]$ is motivated by the fact that this is considered a better metallicity indicator than $[\mathrm{Fe} / \mathrm{H}]$ when comparing stellar populations with different $[\alpha / \mathrm{Fe}]$, like GGCs and dwarf galaxies. value derived by McConnachie et al. (2006). In our CMD there are stars other than the two C-stars listed by Battinelli \& Demers (2000) in the region where C-stars are expected to be found. We assumed as C-stars candidates all objects in our CMD with $J-K_{\mathrm{s}}>1.1$ and $K_{\mathrm{s}}<18.6$. As shown in Fig. 2 there are ten objects within this region, the two Battinelli \& Demers (2000) C-stars and eight objects that can be considered good carbon stars candidates. Nonetheless, to confirm them, higher spatial resolution data, possibly taken with the HST/ACS, are required to distinguish them from background galaxies or photometric blends. The more detailed analysis of SagDIG red stars presented in the following sections shows that SOFI near-IR imaging is a very powerful tool for detecting C-stars, but completing the analysis with ACS optical imaging greatly improves the quality and reliability of the conclusions.

In Fig. 3 the near-IR data are combined with the I-band photometry. In the left panel the upper RGB is visible. Lee et al. (1999) found the TRGB at $I_{\mathrm{TRGB}}=20.95 \pm 0.10$. The calibration of our I magnitudes has larger uncertainty, but nevertheless the TRGB position in our CMD is in good agreement with the Lee et al. (1999) measurement. In the right panel, the two-color diagram is presented. The almost vertical sequence of stars with $J-K_{\mathrm{s}} \simeq 0.9$ is populated by foreground Milky Way stars. The red sequence at $J-K_{\mathrm{s}}>1.1$ and $I-K_{\mathrm{s}}>2.2$ is the region where the carbon stars are expected (e.g. Cioni et al. 2004) and in fact the two carbon stars identified by Battinelli \& Demers (2000) are located in this region. We point out that all the other eight red objects are found along this sequence. In particular there is one very red object with $J-K_{\mathrm{s}}=2.9$, which, if confirmed, could be an AGB star enshrouded in an extended envelope of dust ejected by stellar winds. The coordinates and photometry of the candidates carbon stars are listed in Table 3.

During the preparation of this manuscript McConnachie et al. (2006) published an optical study of the stellar content of DDO 210. From the mean I-band magnitude of the stars located in a red clump they derive an average age of $4_{-1}^{+2}$ Gyr for the majority of stars in DDO 210. They also derived a mean 

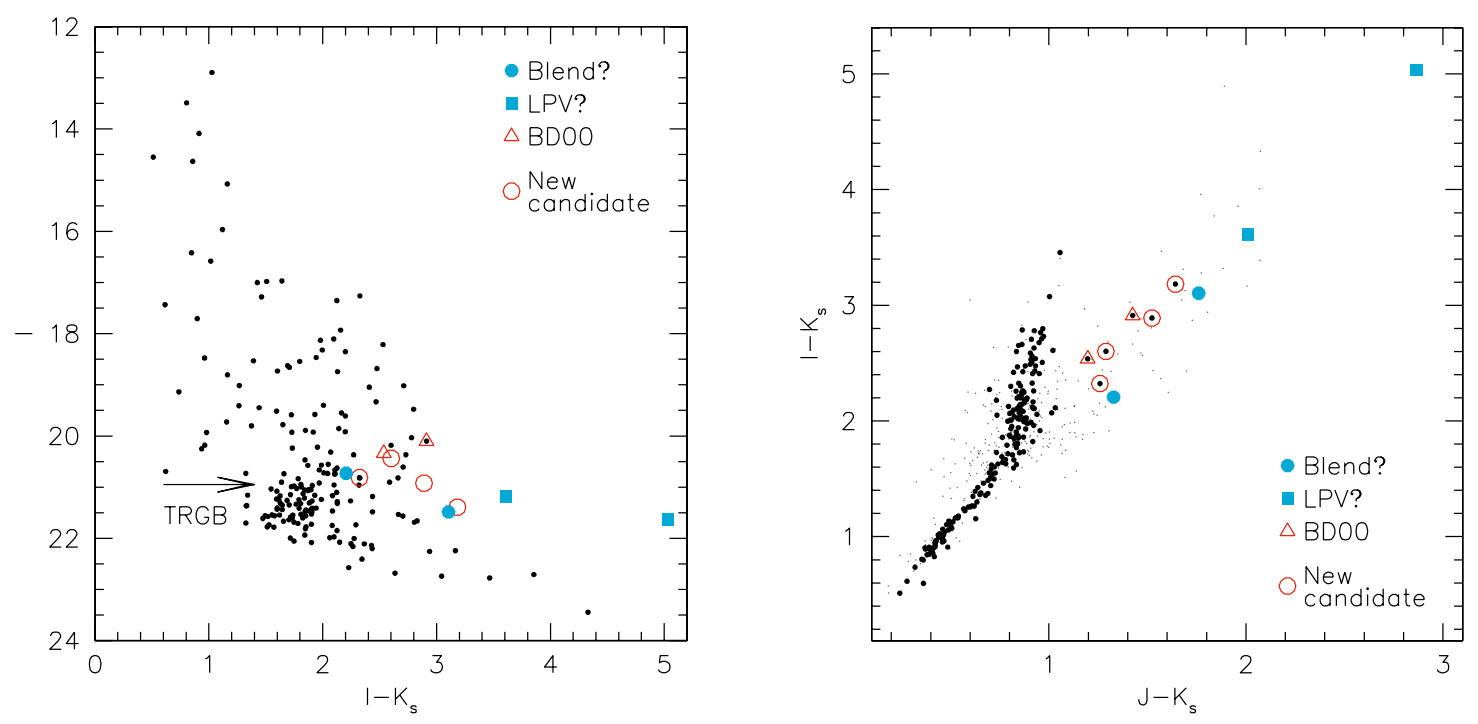

Fig. 3. Left panel: optical - near-IR CMD of DDO 210 stars within $2^{\prime}$ from the center. The outer regions are omitted from this plot for clarity. The arrow shows the position of the TRGB as determined by Lee et al. (1999). One of the two C-stars identified by Battinelli \& Demers (2000), shown as triangles, is located at a greater distance, and is not present in this CMD. The symbols are the same as in Fig. 2. Right panel: the two-color diagram of DDO 210. Larger (darker) dots are stars with $K_{\mathrm{s}}<18.6$.

Table 3. DDO 210 stars with $J-K_{\mathrm{s}}>1.1$ and brighter than $K_{\mathrm{s}}=18.6$. In the last column the cross identification with (Battinelli \& Demers 2000, BD00) carbon stars list is reported. Stars marked with $L P V$ are candidate long period variables, while stars marked with Blue are too blue in optical bands and are likely photometric blends (see text for more details). For candidate LPVs the photometric variation is reported.

\begin{tabular}{ccccccc}
\hline \hline ID & $\delta(2000)$ & $\alpha(2000)$ & $K_{\mathrm{s}}$ & $J-K_{\mathrm{s}}$ & $I-K_{\mathrm{s}}$ & note \\
\hline 1 & $20^{\mathrm{h}} 46^{\mathrm{m}} 47^{\mathrm{s}} .79$ & $-12^{\circ} 50^{\prime} 51^{\prime \prime} \cdot 77$ & 18.38 & 1.76 & 3.10 & Blue \\
2 & $20^{\mathrm{h}} 46^{\mathrm{m}} 46^{\mathrm{s}} .51$ & $-12^{\circ} 52^{\prime} 57^{\prime \prime} .03$ & 18.52 & 1.33 & 2.21 & Blue \\
3 & $20^{\mathrm{h}} 46^{\mathrm{m}} 39.22$ & $-12^{\circ} 50^{\prime} 31^{\prime \prime} 36$ & 17.80 & 1.20 & 2.54 & BD00 C02 \\
4 & $20^{\mathrm{h}} 47^{\mathrm{m}} 06^{\mathrm{s}} .34$ & $-12^{\circ} 50^{\prime} 599^{\prime} \cdot 51$ & 17.84 & 1.29 & 2.60 & \\
5 & $20^{\mathrm{h}} 47^{\mathrm{m}} 04.23$ & $-12^{\circ} 49^{\prime} 54^{\prime \prime} 10$ & 18.21 & 1.64 & 3.18 & \\
6 & $20^{\mathrm{h}} 47^{\mathrm{m}} 01^{\mathrm{s}} .60$ & $-12^{\circ} 52^{\prime} 51^{\prime \prime} \cdot 76$ & 18.03 & 1.52 & 2.89 & \\
7 & $20^{\mathrm{h}} 46^{\mathrm{m}} 53^{\mathrm{s}} .92$ & $-12^{\circ} 50^{\prime} 37^{\prime \prime} 68$ & 16.59 & 2.86 & 5.03 & LPV? $\Delta I=-1.59$ \\
8 & $20^{\mathrm{h}} 46^{\mathrm{m}} 53^{\mathrm{s}} .24$ & $-12^{\circ} 50^{\prime} 25^{\prime \prime} \cdot 31$ & 17.58 & 2.01 & 3.61 & LPV? $\Delta I=0.45$ \\
9 & $20^{\mathrm{h}} 46^{\mathrm{m}} 52^{\mathrm{s}} .87$ & $-12^{\circ} 50^{\prime} 25^{\prime \prime} 62$ & 17.19 & 1.43 & 2.91 & BD00 C03 \\
10 & $20^{\mathrm{h}} 46^{\mathrm{m}} 52^{\mathrm{s}} .50$ & $-12^{\circ} 50^{\prime} 10^{\prime} 68$ & 18.49 & 1.26 & 2.32 & \\
\hline
\end{tabular}

age-corrected metallicity of $[\mathrm{Fe} / \mathrm{H}]=-1.3$, which is much higher than the value $[\mathrm{Fe} / \mathrm{H}]=-1.9$ derived by Lee et al. (1999) and reported in Table 1. These results suggest that a significant population of carbon-rich AGB giants is expected to be present in this galaxy. In their CMDs they identify a bright asymptotic giant branch population. This further confirms the reality of our carbon-star candidates.

It is acknowledged that most, if not all, of the carbon giants vary on long time-scales (e.g. Groenewegen 2004; Raimondo et al. 2005). We therefore tried to search for variable star candidates by comparing the $I$-band photometry presented in this work with that of McConnachie et al. (2006). With only two epochs (separated by 734 days) it is possible to give only a lower limit to the number of long period variable (LPV) stars. It is however encouraging that two out of eight new carbon-star candidates are also candidate LPV stars. The differences in magnitude between the two epochs are reported in Table 3. We note that these are lower limits to the variability amplitudes and that they are significantly larger than the combined photometric errors at the corresponding magnitude. Obviously to confirm the variable nature of these stars, and to be able to reliably estimate the number of LPVs in this galaxy, more observations are necessary. The two carbon-stars previously identified by Battinelli \& Demers (2000) do not present variability between the two epochs within the errors of photometric measurements. Finally we note that the amplitude of LPV light curves increases at increasing colors (e.g. Raimondo et al. 2005) and in fact our two candidate LPVs are the two reddest candidate C-stars.

Finally we checked the position of all ten carbon star candidates in our $I-K_{\mathrm{s}}$ vs. $I$ CMD (Fig. 3 ) and in optical CMDs using McConnachie et al. (2006) data. Eight of them, including the two candidate LPVs and the two stars classified as C-stars by Battinelli \& Demers (2000), are in the expected location for AGB stars. The other two, marked in Fig. 2 (filled circles) and Table 3 ("Blue"), are too blue in optical bands (e.g. in $B-V$ ). They are similar to the three blue objects found among SagDIG C-stars candidates (see next sections), with blue colors in the optical and red colors in the near-IR, and thus it is possible that they could be blends of two or more unresolved stars or background galaxies.

Battinelli \& Demers (2000) claim that their C-star candidate in DDO 210 may be unusually bright. In Fig. 2 our CMD is compared with the mean color-magnitude relation for C-stars derived by Totten et al. (2000) as a fit to the photometry of a sample of carbon stars from Milky Way satellite galaxies. We assumed the distance modulus $(m-M)_{0}=20.89$ obtained by Lee et al. (1999), but we used also the longer distance $(m-M)_{0}=25.15$ derived by McConnachie et al. (2006). In both cases the C-stars 


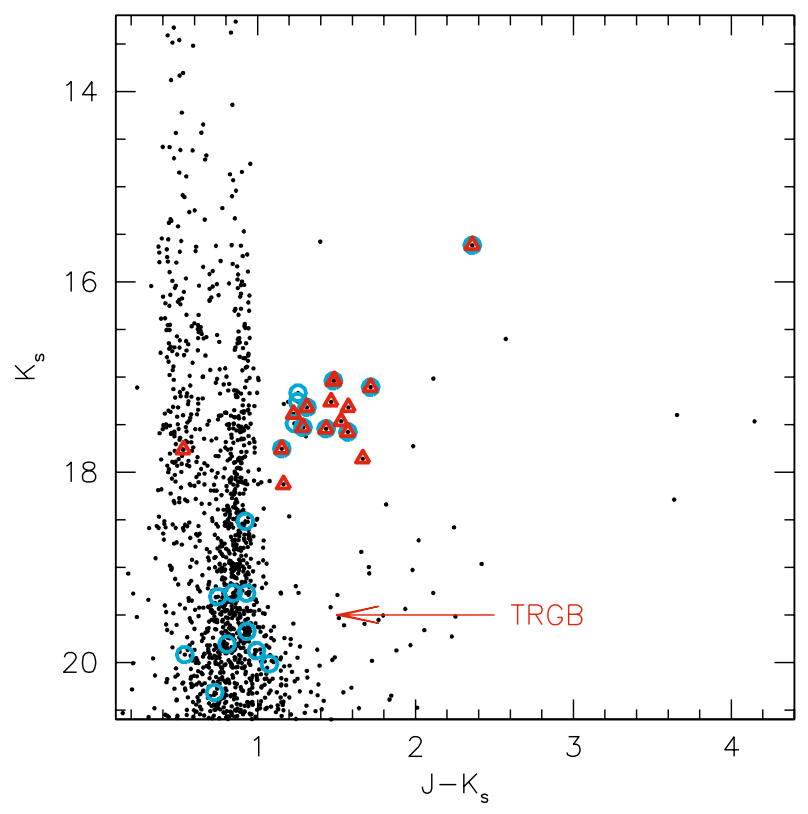

Fig. 4. The near-IR CMD of SagDIG and the carbon stars identified by Cook (1987) (circles) and Demers \& Battinelli (2002) (triangles). Some of the carbon stars from Cook (1987) are fainter than the TRGB, shown by the arrow.

detected by Battinelli \& Demers (2000) appear to be slightly brighter than the Totten et al. (2000) relation. Interestingly our new candidates bring the C-star population of DDO 210 in agreement with the Totten et al. (2000) locus.

Summarizing, the number of bona fide carbon stars in DDO is nine. This includes three stars previously known from Battinelli \& Demers (2000), and six new candidates discovered in this work that have both optical and near-IR colors consistent with carbon giants. Two additional red objects are probable blends or unresolved background galaxies.

\subsection{SagDIG}

The CMD of SagDIG is shown in Fig. 4. Stellar objects were selected using the SHARP parameter provided by ALLFRAME, as described in Sect. 5.1. Also in this case the two almost vertical sequences at $J-K_{\mathrm{s}} \sim 0.6$ and $J-K_{\mathrm{s}} \sim 0.9$, populated by foreground Galactic stars, are clearly visible (e.g. Nikolaev $\&$ Weinberg 2000).

In order to estimate the contribution of foreground Galactic stars we compared the CMDs shown in Fig. 5, obtained from the outer and the inner fields, but only from our observations (August 2003). In this way we compare data obtained in exactly the same way, and observed under similar seeing conditions, so that the completeness and photometric errors in the two CMDs are similar. The CMDs in Fig. 5 are obtained with the same SHARP selection defined to plot Fig. 4, which is obtained combining 1998 and 2003 observations. The quality of photometry in Fig. 5 is clearly lower, and in particular, for the same star, the SHARP value is higher in the photometry obtained from 2003 observations alone. For this reason some stars which are present in Fig. 4 are absent in Fig. 5. The box in Fig. 5 corresponds to the region occupied by C-stars which is defined and discussed in detail below. No object is seen inside the box in the outer field apart from two objects at the faint limit of the box, which are likely background galaxies. Moreover a statistical analysis showed that the star counts in both CMDs (excluding the C-stars

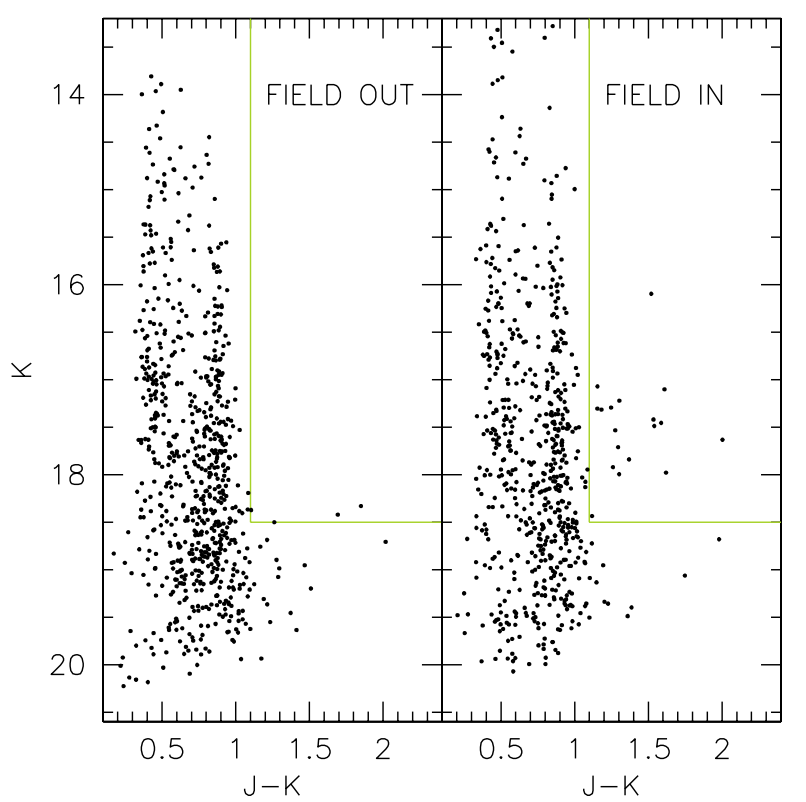

Fig. 5. CMDs of the outer (left) and inner (right) SagDIG fields from August 2003 observations. The box represents the region occupied by C-stars.

region) are identical. Adopting a distance modulus of 25.10 and $[\mathrm{M} / \mathrm{H}]=[\mathrm{Fe} / \mathrm{H}]=-2.0$, using the relation of Valenti et al. (2004) and the Rieke \& Lebofsky (1985) reddening law, we derived that the expected magnitude for the tip of the red giant branch is $K_{\mathrm{s}}=19.5$, which is close to the limiting magnitude in Fig. 5 . We therefore conclude that all the stars brighter than $K_{\mathrm{s}}=19.5$ and bluer than $J-K_{\mathrm{s}}=1.1$ are most probably foreground stars and that all the stars redder than this limit are candidate $C$-stars belonging to SagDIG. The only possible exception is the presence of AGB oxygen rich M-stars of SagDIG, which are expected to have magnitudes brighter than the RGB tip, but colors similar to that of foreground stars (Nikolaev \& Weinberg 2000). However, given the low metallicity of SagDIG we expect a high $\mathrm{C} / \mathrm{M}$ ratio and therefore a very small number of M-stars. This is confirmed by the similarity of the luminosity function (LF) of the inner and outer fields in the color range occupied by M-stars.

The CMD in Fig. 4 obtained combining all the SagDIG data (August $1998+$ August 2003) is clearly deeper and extends to $K_{\mathrm{s}}=21$. The contamination from foreground stars is however high, and it is impossible to distinguish the RGB of SagDIG.

We combined our data with the HST/ACS photometry of Momany et al. (2005), transforming the ACS magnitudes from the HST system to that defined by Stetson (2000) (which is equivalent to the Landoldt standard system) using the equation given by Rejkuba et al. (2005):

$V-F 606 W=0.222(V-I)+0.072$

$I-F 814 W=-0.042(V-I)+0.130$.

With a wider baseline it is possible to separate the sequence of foreground stars and the RGB of SagDIG, which is visible in the right panel of Fig. 6. The foreground stars sequences, which are very narrow in $J-K_{\mathrm{s}}$ color are much broader in $V-K_{\mathrm{s}}$ and extend up to $V-K_{\mathrm{s}} \sim 7$ (see also Fig. 7). In the upper right panel of Fig. 6 we show the $V-K_{\mathrm{s}}$ color distribution of stars with $20.5<K_{\mathrm{s}}<19.5$. The detection of the RGB is clearly visible as an overdensity at $V-K_{\mathrm{s}} \sim 3.2$. The color of RGB stars is consistent with the RGB fiducial lines of Milky Way globular clusters taken from Valenti et al. (2004) of M $15([\mathrm{Fe} / \mathrm{H}]=-2.12$, 

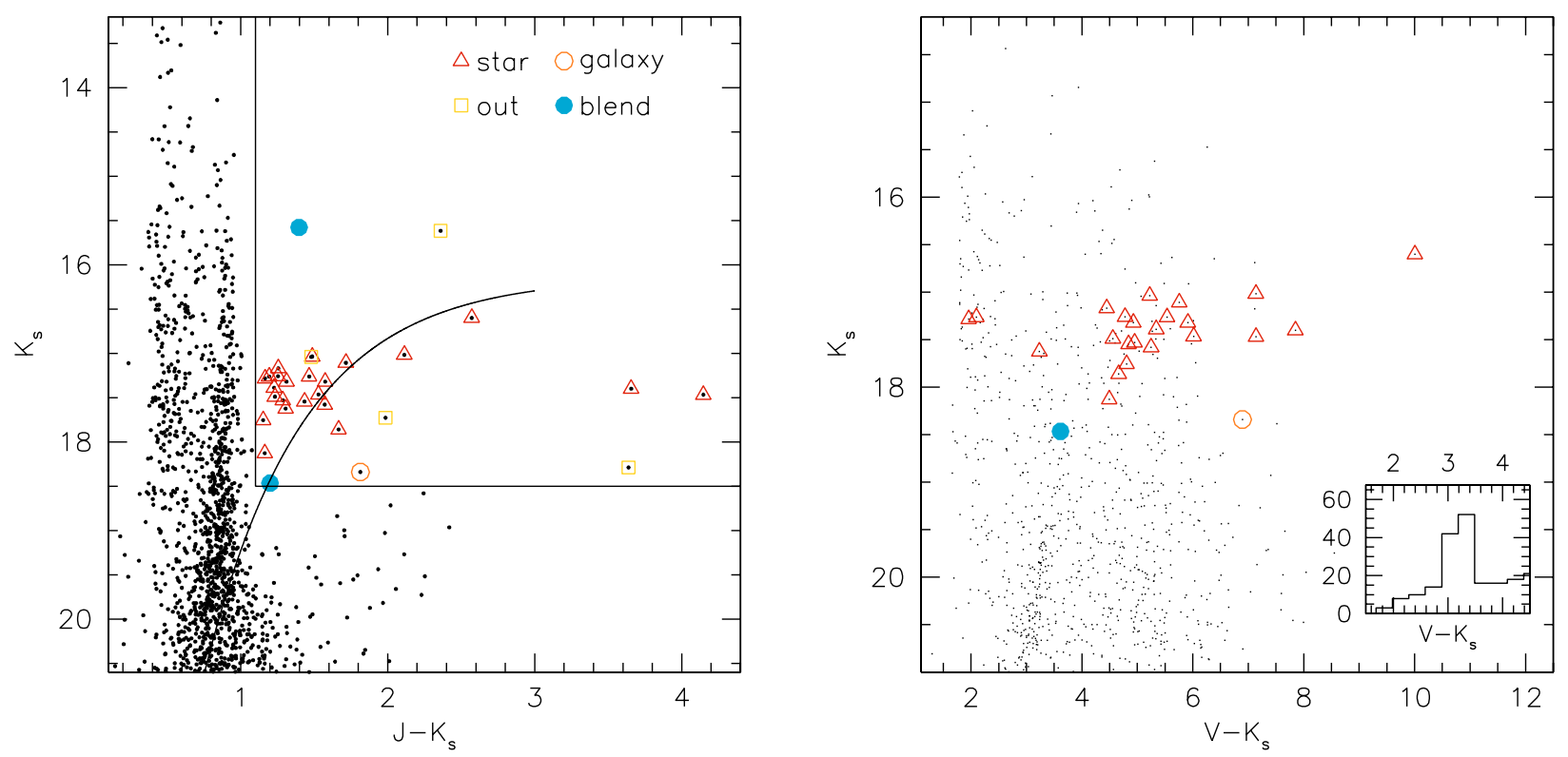

a)
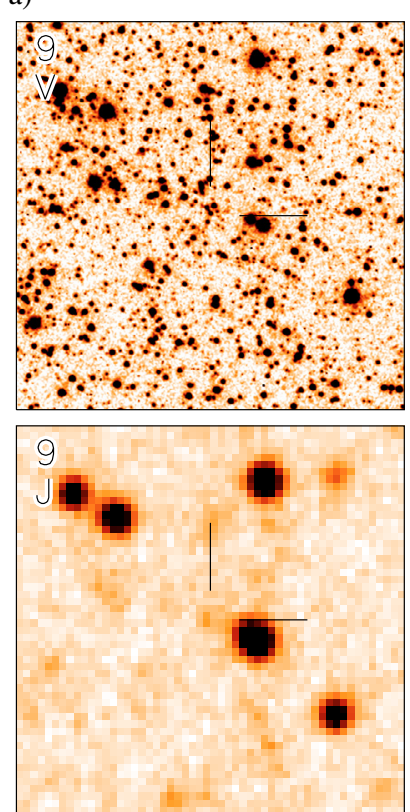

c)

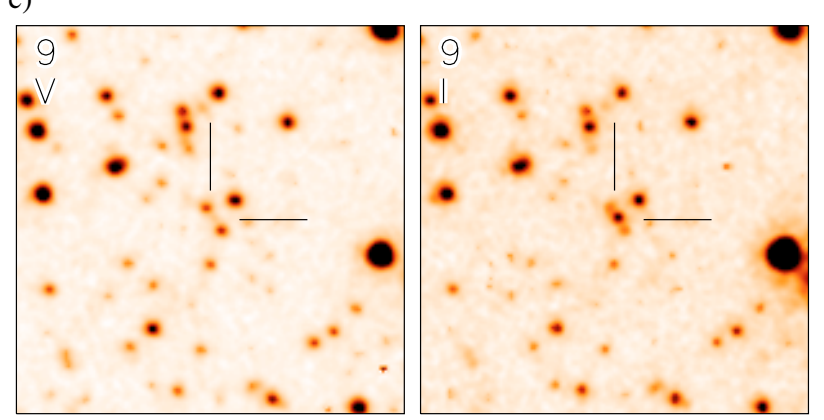

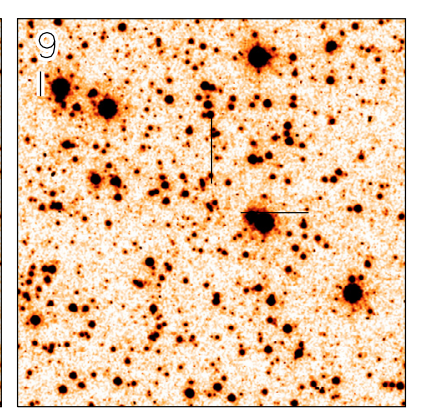

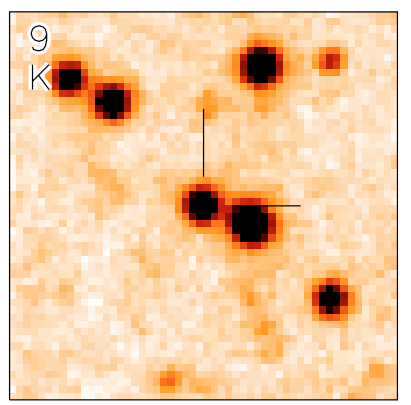

b)
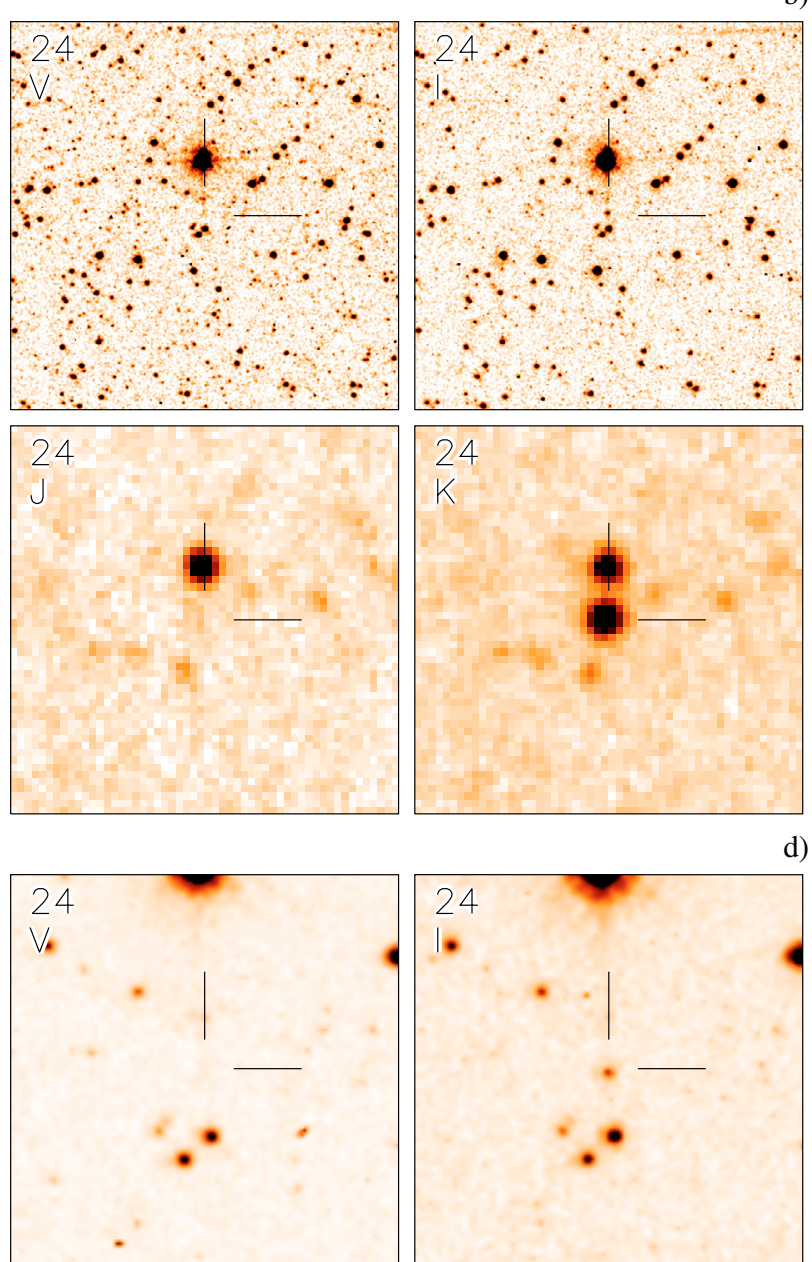

d)

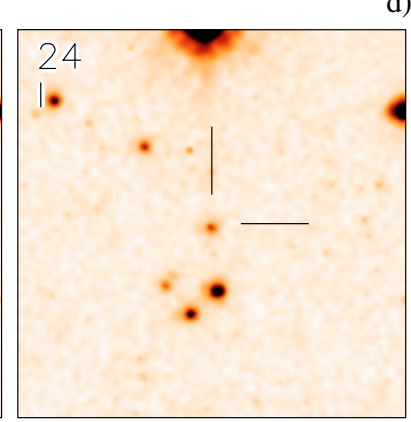

Fig. 6. Upper panels: classification of the red stars in our catalogue, based on HST/ACS images (Momany et al. 2005). We plotted stars outside the field of view of the ACS observations as open squares. In the $K_{\mathrm{s}}, J-K_{\mathrm{s}} \mathrm{CMD}$ we show the mean carbon star color-magnitude relation from Totten et al. (2000) and the box we used to define C-star candidates. The inset in the right panel shows the detection of the RGB in the $V-K_{\mathrm{s}}$ color distribution of stars with $20.5<K_{\mathrm{s}}<19.5$. Lower panels, a) and b): Zoom in the regions of the two reddest stars (number 9 and 24) of SagDIG. Upper panels are HST/ACS $V$ and $I$ images and lower panels are $J$ and $K_{\mathrm{s}}$ SOFI images. The images show areas of $15^{\prime \prime} \times 15^{\prime \prime}$ centered on the red star. North is up and East is left. The two lowest panels, c) and d), are a further enlargement of ACS images $\left(33^{\prime \prime} 5 \times 3\right.$ ". 5$)$. 
$[\mathrm{M} / \mathrm{H}]=-1.91)$ and $\mathrm{M} 30([\mathrm{Fe} / \mathrm{H}]=-1.91,[\mathrm{M} / \mathrm{H}]=-1.71)$, confirming the low metallicity of SagDIG (Saviane et al. 2002; Momany et al. 2005). Finally we note that the position of the RGB tip is in good agreement with the value of $K_{\mathrm{s}}=19.5$ expected from the Valenti et al. (2004) relations.

\section{SagDIG carbon stars}

In Fig. 4 we show the carbon stars identified by Cook (1987) and Demers \& Battinelli (2002). Near-IR photometry confirms that six of them are fainter than the RGB tip $\left(K_{\mathrm{s}}=19.5\right)$. From our photometry it is difficult to determine their origin, but we note that the HST photometry of Momany et al. (2005) demonstrates that three of them have colors similar to RGB stars, and are likely to belong to an older AGB population while the others could be Galactic dwarf C-stars. We note that there are also four stars detected by Cook (1987) and one from Demers \& Battinelli (2002) that are brighter than $K_{\mathrm{s}}=19.5$ and bluer than $J-K_{\mathrm{s}}=1.1$, but again in this case it is impossible to distinguish them from foreground stars.

In the upper left panel of Fig. 6 our CMD is compared with the main locus of C-stars in LG galaxies from Totten et al. (2000), scaled to the distance of SagDIG. This C-star fiducial suggests that all stars brighter than $K_{\mathrm{s}}=19.5$ and redder than $J-K_{\mathrm{s}}=1.1$ are likely SagDIG C-stars. To minimize contamination by unresolved galaxies and stellar blends in our selection we assume a magnitude limit of $K_{\mathrm{s}}=18.5$ for C-stars. We expect that the red objects at $J-K_{\mathrm{s}} \sim 2$ and fainter than our selection, are most likely background galaxies or stellar blends, because they are too faint to be C-stars compared to Totten et al. (2000) locus.

Below we describe in detail the 30 candidate C-stars belonging to SagDIG listed in Table 4, defined using the following criteria: $J-K_{\mathrm{s}}>1.1$ and $K_{\mathrm{s}}<18.5$. Firstly we performed a visual inspection of all objects in the ACS images. The results are reported in Col. 6 of Table 4 and in Fig. 6. Four of the C-star candidates fall outside the ACS FOV, one is clearly a background galaxy, two have shapes that could be associated with irregular background galaxies or photometric blends. All other objects appear as real stars. We note that all of our six objects redder than $J-K_{\mathrm{s}}=2$, except for the bright source with $K_{\mathrm{s}} \simeq 15.5$, are missed by Cook (1987) and Demers \& Battinelli (2002).

We searched for LPV stars comparing ACS I-band photometry, transformed to standard system according to Eq. (3), with Demers \& Battinelli (2002) I-band photometry of C-star candidates. We detected three stars with significant variability, reported in the last column of Table 4. As in the case of DDO 210 our candidate LPVs are among the reddest stars, in agreement with the color-amplitude relation for LPV stars (Raimondo et al. 2005).

There are also three stars, namely number 6,7 and 12 , with a near-IR color typical of C-stars, but not identified by Cook (1987) or Demers \& Battinelli (2002). Suspiciously, all three stars have optical colors bluer than normal C-stars, i.e. they are the three bluest stars among our AGB candidates in the right panel of Fig. 6, the only ones with $V-K_{\mathrm{s}}<3.5$. The optical colors of these three stars are confirmed by the EMMI photometry of Momany et al. (2002). In the appendix, available in the online version of this paper, we discuss in further detail the nature of these three objects. Their SED is compatible with a simple model obtained summing the SED of a blue and a red star. The most reliable hypothesis is that they are a photometric blends rather than binary systems. The two bluest objects (number 7 and 12) could alternatively be high redshift galaxies.

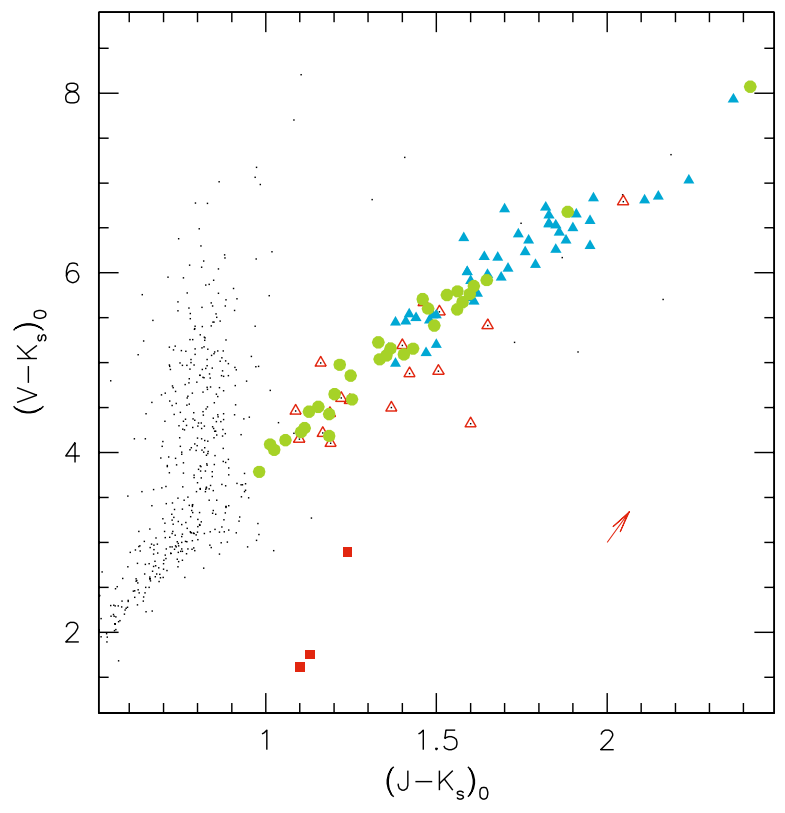

Fig. 7. Two-color diagram of SagDIG stars (black dots) brighter than $K_{\mathrm{s}}=20$ together with Bergeat et al. (2001) Galactic C-stars (large filled blue triangles) and the carbon stars of Fornax (Gullieuszik et al. 2007) (large filled green circles). The open red triangles are the SagDIG C-star candidates, while squares are the three peculiar objects likely not $\mathrm{C}$-stars. The arrow represents the reddening vector toward SagDIG, assuming $E_{(B-V)}=0.12$.

As an example, in the lower panels of Fig. 6 we show a zoom of SOFI and ACS images in the regions of the two reddest stars of SagDIG. As can be seen they are very bright in the $K_{\mathrm{s}}$ images and barely detectable in $J$. Due to the high sensitivity and deep exposure of the ACS images they are clearly visible in the $I$ frames even if the flux in the $I$-band is lower than in $J$, but are extremely faint in $V$. Moreover, especially for star number 9 , the highest sensitivity and spatial resolution of the ACS could resolve in high detail stars that are blended on the SOFI images. In this particular case this is not a great problem because the unresolved stars are extremely faint and do not affect the near-IR measurement significantly. Note that we made a visual inspection of all 30 red stars of SagDIG and found that only two suffer from relevant blending problems.

\subsection{Two-color diagram}

In Fig. 7 the $\left(J-K_{\mathrm{s}}\right)$ vs. $\left(V-K_{\mathrm{s}}\right)$ two-color diagram is shown. The red carbon stars (open triangles) define a quite narrow sequence, while field stars are well separated and lie on an almost vertical sequence at $\left(J-K_{\mathrm{s}}\right) \simeq 0.8$ and $\left(V-K_{\mathrm{s}}\right)>3.5$. On the same diagram we also show the C-stars of Fornax dSph (Gullieuszik et al. 2007) and the galactic C-stars from Bergeat et al. (2001). In Fig. 8 SagDIG stars are shown in the $\left(J-K_{\mathrm{s}}\right)$ vs. $\left(I-K_{\mathrm{s}}\right)$ diagram, together with the carbon stars of Fornax (Gullieuszik et al. 2007) and the carbon stars of the two Magellanic Clouds extracted from the DENIS DCMC catalogue (Cioni et al. 2000).

The metallicities of the considered systems span a wide range $($ SagDIG $[\mathrm{M} / \mathrm{H}] \sim-2.0, \mathrm{SMC}[\mathrm{M} / \mathrm{H}] \sim-0.8, \mathrm{LMC}$ $[\mathrm{M} / \mathrm{H}] \sim-0.6$, Fornax $[\mathrm{M} / \mathrm{H}] \sim-0.9$. Stars from Bergeat et al. (2001) have a wide range of metallicity as they are drawn from the Galactic sample), but C-star sequences from all these systems overlap on a well-defined common straight line. 
Table 4. The red stars selected with $J-K_{\mathrm{s}}>1.1$ and $K_{\mathrm{s}}<18.5$. Columns 7 and 8 give the identifications from Cook (1987, C87) and Demers \& Battinelli (2002, DB02). The notes in Col. 6 refer to the classification of the objects based on visual inspection of $V$ and $I$-band HST/ACS images (Momany et al. 2005): 0: galaxy; 1: star; 2: outside the ACS FOV; 3: blend. Stars marked as Blue are too blue in optical bands (see text for details). Last column reports the detection of significant $I$-band variability for Demers \& Battinelli (2002) C-star candidates.

\begin{tabular}{|c|c|c|c|c|c|c|c|c|}
\hline$\overline{\mathrm{ID}}$ & $\delta(2000)$ & $\alpha(2000)$ & $K_{\mathrm{s}}$ & $J-K_{\mathrm{s}}$ & Note & C87 & $\overline{\mathrm{DB} 02}$ & $\overline{\Delta I}$ \\
\hline 1 & $19^{\mathrm{h}} 30^{\mathrm{m}} 08^{\mathrm{s}} .14$ & $-17^{\circ} 40^{\prime} 00^{\prime} \cdot 84$ & 17.041 & 1.480 & 2 & 1 & 2 & \\
\hline 2 & $19^{\mathrm{h}} 30^{\mathrm{m}} 06^{\mathrm{s}} .25$ & $-17^{\circ} 40^{\prime} 54^{\prime \prime} 66$ & 15.616 & 2.360 & 2 & 2 & 5 & \\
\hline 3 & $19^{\mathrm{h}} 30^{\mathrm{m}} 05^{\mathrm{s}} .17$ & $-17^{\circ} 41^{\prime} 42^{\prime \prime} \cdot 79$ & 17.389 & 1.227 & 1 & - & 9 & \\
\hline 4 & $19^{\mathrm{h}} 30^{\mathrm{m}} 04^{\mathrm{s}} .17$ & $-17^{\circ} 40^{\prime} 30^{\prime} .27$ & 17.754 & 1.153 & 1 & 4 & 8 & \\
\hline 5 & $19^{\mathrm{h}} 30^{\mathrm{m}} 04^{\mathrm{s}} .08$ & $-17^{\circ} 40^{\prime} 15^{\prime \prime} .65$ & 18.340 & 1.814 & 0 & - & - & \\
\hline 6 & $19^{\mathrm{h}} 30^{\mathrm{m}} 03^{\mathrm{s}} .25$ & $-17^{\circ} 41^{\prime} 33^{\prime \prime} .80$ & 17.624 & 1.306 & 1, Blue & - & - & \\
\hline 7 & $19^{\mathrm{h}} 30^{\mathrm{m}} 02^{\mathrm{s}} .02$ & $-17^{\circ} 40^{\prime} 19^{\prime} .58$ & 17.262 & 1.194 & 1, Blue & - & - & \\
\hline 8 & $19^{\mathrm{h}} 30^{\mathrm{m}} 02^{\mathrm{s}} .01$ & $-17^{\circ} 42^{\prime} 05^{\prime \prime} .77$ & 17.036 & 1.487 & 1 & - & 3 & \\
\hline 9 & $19^{\mathrm{h}} 30^{\mathrm{m}} 01^{\mathrm{s}} .75$ & $-17^{\circ} 40^{\prime} 52^{\prime \prime} .79$ & 17.400 & 3.656 & 1 & - & - & \\
\hline 10 & $19^{\mathrm{h}} 30^{\mathrm{m}} 01^{\mathrm{s}} .68$ & $-17^{\circ} 40^{\prime} 04^{\prime \prime} .29$ & 17.169 & 1.256 & 1 & 5 & - & \\
\hline 11 & $19^{\mathrm{h}} 30^{\mathrm{m}} 01^{\mathrm{s}} .67$ & $-17^{\circ} 41^{\prime} 06^{\prime \prime} .72$ & 17.465 & 1.529 & 1 & - & 10 & 0.31 \\
\hline 12 & $19^{\mathrm{h}} 30^{\mathrm{m}} 01^{\mathrm{s}} .12$ & $-17^{\circ} 39^{\prime} 59^{\prime} .40$ & 17.284 & 1.166 & 1, Blue & - & - & \\
\hline 13 & $19^{\mathrm{h}} 30^{\mathrm{m}} 01^{\mathrm{s}} .01$ & $-17^{\circ} 40^{\prime} 52^{\prime \prime} .74$ & 17.319 & 1.312 & 1 & 6 & 1 & \\
\hline 14 & $19^{\mathrm{h}} 30^{\mathrm{m}} 00^{\mathrm{s}} .68$ & $-17^{\circ} 41^{\prime} 00^{\prime} .^{\prime} 69$ & 17.017 & 2.113 & 1 & - & - & \\
\hline 15 & $19^{\mathrm{h}} 30^{\mathrm{m}} 00^{\mathrm{s}} .29$ & $-17^{\circ} 41^{\prime} 02^{\prime \prime} .92$ & 17.107 & 1.716 & 1 & 8 & 15 & -0.48 \\
\hline 16 & $19^{\mathrm{h}} 30^{\mathrm{m}} 00^{\mathrm{s}} .23$ & $-17^{\circ} 40^{\prime} 54^{\prime \prime} 61$ & 17.579 & 1.572 & 1 & 9 & 16 & \\
\hline 17 & $19^{\mathrm{h}} 30^{\mathrm{m}} 00^{\mathrm{s}} .07$ & $-17^{\circ} 41^{\prime} 35^{\prime \prime} .29$ & 17.859 & 1.666 & 1 & - & 4 & 0.53 \\
\hline 18 & $19^{\mathrm{h}} 29^{\mathrm{m}} 59^{\mathrm{s}} .79$ & $-17^{\circ} 41^{\prime} 22^{\prime \prime} .61$ & 18.465 & 1.200 & 3 & - & - & \\
\hline 19 & $19^{\mathrm{h}} 29^{\mathrm{m}} 59^{\mathrm{s}} .77$ & $-17^{\circ} 41^{\prime} 05^{\prime \prime} .14$ & 16.600 & 2.572 & 1 & - & - & \\
\hline 20 & $19^{\mathrm{h}} 29^{\mathrm{m}} 59^{\mathrm{s}} .76$ & $-17^{\circ} 40^{\prime} 34^{\prime \prime} .23$ & 17.489 & 1.233 & 1 & 11 & - & \\
\hline 21 & $19^{\mathrm{h}} 29^{\mathrm{m}} 57^{\mathrm{s}} .95$ & $-17^{\circ} 40^{\prime} 17^{\prime \prime} .54$ & 15.578 & 1.397 & 3 & - & - & \\
\hline 22 & $19^{\mathrm{h}} 29^{\mathrm{m}} 57^{\mathrm{s}} .55$ & $-17^{\circ} 40^{\prime} 41^{\prime \prime} .96$ & 18.128 & 1.164 & 1 & - & 13 & \\
\hline 23 & $19^{\mathrm{h}} 29^{\mathrm{m}} 56^{\mathrm{s}} .34$ & $-17^{\circ} 40^{\prime} 47^{\prime \prime} .41$ & 17.261 & 1.466 & 1 & - & 11 & \\
\hline 24 & $19^{\mathrm{h}} 29^{\mathrm{m}} 56^{\mathrm{s}} .08$ & $-17^{\circ} 41^{\prime} 21^{\prime \prime} .50$ & 17.467 & 4.147 & 1 & - & - & \\
\hline 25 & $19^{\mathrm{h}} 29^{\mathrm{m}} 55^{\mathrm{s}} .24$ & $-17^{\circ} 40^{\prime} 22^{\prime \prime} 79$ & 17.529 & 1.287 & 1 & 16 & 7 & \\
\hline 26 & $19^{\mathrm{h}} 29^{\mathrm{m}} 55^{\mathrm{s}} .15$ & $-17^{\circ} 40^{\prime} 40^{\prime} .79$ & 17.259 & 1.254 & 1 & 17 & - & \\
\hline 27 & $19^{\mathrm{h}} 29^{\mathrm{m}} 52^{\mathrm{s}} .90$ & $-17^{\circ} 40^{\prime} 32^{\prime \prime} .86$ & 17.545 & 1.434 & 1 & 19 & 6 & \\
\hline 28 & $19^{\mathrm{h}} 29^{\mathrm{m}} 52^{\mathrm{s}} .90$ & $-17^{\circ} 41^{\prime} 39^{\prime} .54$ & 17.319 & 1.575 & 1 & - & 14 & \\
\hline 29 & $19^{\mathrm{h}} 29^{\mathrm{m}} 51^{\mathrm{s}} .64$ & $-17^{\circ} 39^{\prime} 44^{\prime \prime} .31$ & 18.289 & 3.638 & 2 & - & - & \\
\hline 30 & $19^{\mathrm{h}} 29^{\mathrm{m}} 49^{\mathrm{s}} .21$ & $-17^{\circ} 41^{\prime} 03^{\prime \prime} 05$ & 17.727 & 1.985 & 2 & - & - & \\
\hline
\end{tabular}

\section{2. $L F$ and SFH}

The number of $\mathrm{C}$ stars present in SagDIG is sufficient to derive from their $K_{\mathrm{s}}$ magnitude distribution, an indication about the mean metallicity and age of the underlying stellar population using the same criterion adopted by Cioni et al. (2006a). Probable galaxies and blends (see note in Table 4) have been excluded from this analysis. We note that three objects among our C-star candidates (number 6, 7 and 12) have peculiar optical colors, and we cannot conclude whether they are C-stars blended with blue stars or foreground galaxies. We therefore studied the $K_{\mathrm{s}}$-band LF obtained from the whole sample of $27 \mathrm{C}$-stars candidates, but the analysis was repeated excluding the three peculiar objects.

The observed $K_{\mathrm{s}}$-band distributions of the $\mathrm{C}$ stars is shown in Fig. 9. When all 27 stars are considered, the LF peaks around $K_{\mathrm{s}}=17.3$ and is asymmetric. We note that the three peculiar objects populate the peak of the LF, and excluding them significantly changes the peak. The two $K_{\mathrm{s}}$-band LFs have been compared with theoretical distributions created as in Cioni et al. (2006a) using up-to-date stellar evolutionary tracks and a population synthesis code (TRILEGAL; Girardi et al. 2005). The code randomly generates stars following a given star formation rate (SFR), age-metallicity relation and initial mass function. The intrinsic stellar properties are interpolated over a large grid of stellar evolutionary tracks, based on Bertelli et al. (1994) and Girardi et al. (2003) for massive stars, Girardi et al. (2000) for low- and intermediate-mass stars, and complemented with the grids of thermally pulsing AGB tracks described by Marigo et al. (1999, 2003). Near-IR magnitudes were simulated from bolometric magnitudes and photometric errors were also included. More details about the construction and properties of the models are given in Cioni et al. (2006a) while the isochrones used in this study are available at http://pleiadi.oapd.inaf.it. Five different cases for metallicity and SFR have been adopted resulting in 25 possible combinations which in turn have been compared with the observed distribution of $\mathrm{C}$ stars. The metallicity values are expressed in terms of $Z=0.0005,0.001,0.004,0.008$ and 0.016. The SFR is described as $\psi(t) \propto \exp (t / \alpha)$, where $t$ is the stellar age, and $\alpha$ a free parameter. We have considered values for $\alpha$ of: $-5,-2,1000,5$ and 2 which correspond to a meanage of all stars of 2, 3.9, 6.3, 8.7 and 10.6 Gyr respectively. The comparison between observed and theoretical $K_{\mathrm{s}}$-band distributions has been evaluated using the statistical $\chi^{2}$ test where only bins containing more than one source have been considered.

Figure 10 shows the most probable metallicity at a given age for the stellar population of SagDIG. In the upper panel we show the probability obtained considering all 27 C-star candidates, while the lower panel shows the result obtained excluding the three peculiar objects. The point corresponding to the highest probability in the upper panel indicates that the overall population is young (on average $4 \mathrm{Gyr}$ old) and metal-poor (at least $[\mathrm{Fe} / \mathrm{H}]<-1.3$ dex assuming $Z_{\odot}=0.02$ ). However, these numbers need to be taken with care due to the limited sample of C-stars and the magnitude binning adopted. When the three peculiar objects are not considered, the model with the highest 


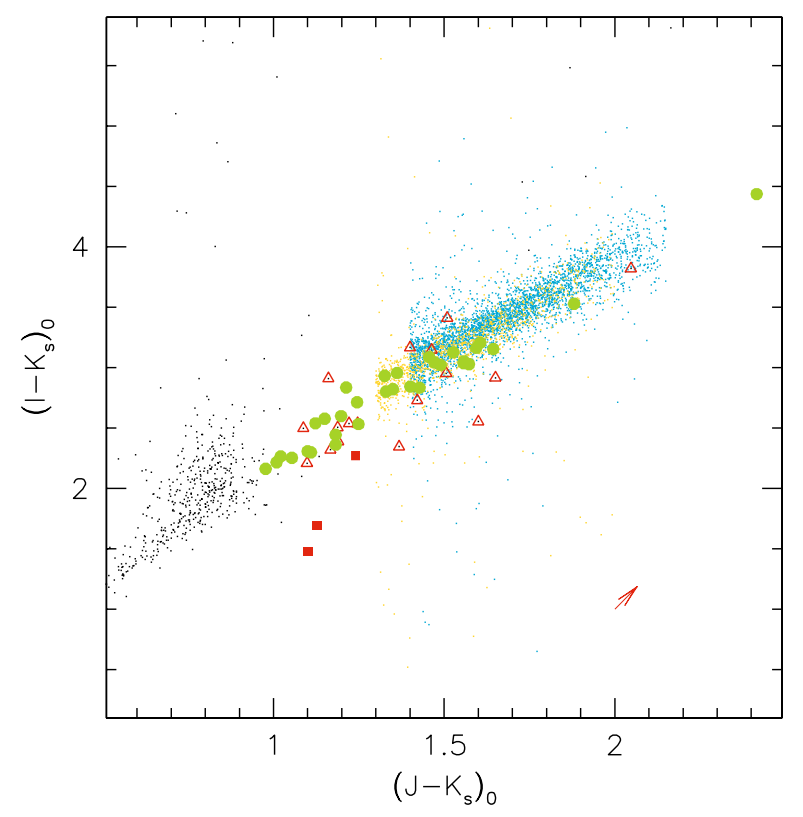

Fig. 8. Two-color diagram of SagDIG stars brighter than $K_{\mathrm{s}}=20$. Small points are C-stars of LMC (blue) and SMC (yellow). Filled green circles are C-stars of Fornax. The open red triangles are the SagDIG C-star candidates, while squares are the three peculiar objects likely not C-stars. The arrow represents the reddening vector toward SagDIG, assuming $E_{(B-V)}=0.12$.

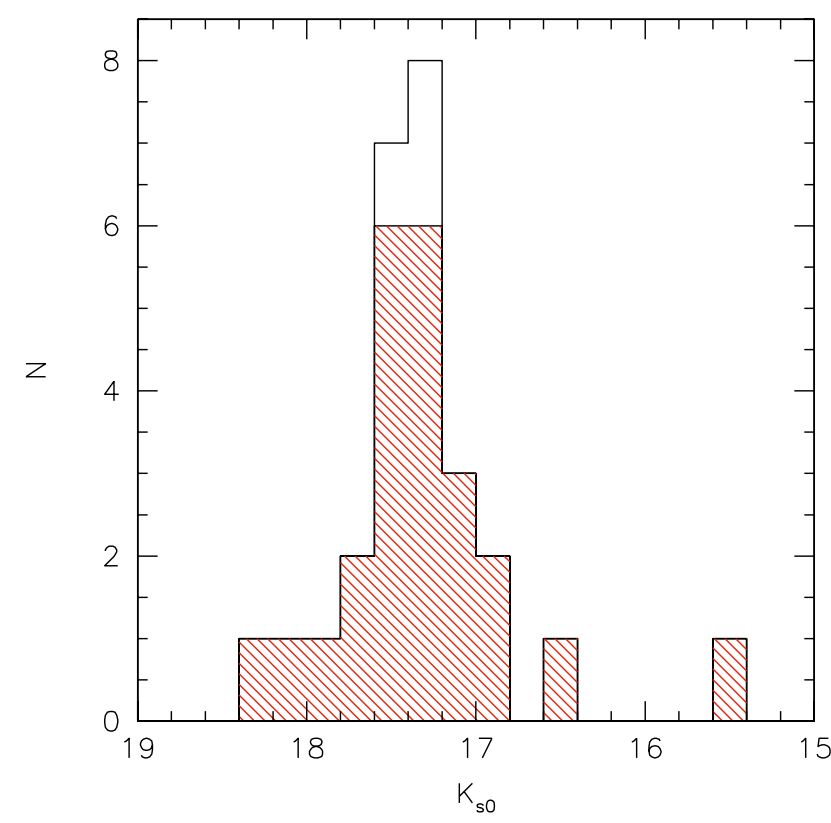

Fig. 9. Observed $K_{s 0}$ de-reddened magnitude distribution of C-stars in SagDIG. Bins are of $0.2 \mathrm{mag}$. The dashed histogram is obtained excluding the three peculiar objects with blue optical colors.

probability turns out to be more metal-poor $(Z=0.0005$, corresponding to $[\mathrm{Fe} / \mathrm{H}]=-1.6)$. In order to have an estimate of the significance of our results and of the error-bars to be associated with the points in Fig. 10, we applied the JACKNIFE method to our sample. We repeated our analysis five times, removing in turn an object from each of the bins with more than one star in the histogram in Fig. 9. This resulted in a large spread in the probabilities corresponding to models with $Z>0.0005$. On the other hand for $Z=0.0005$ models, a much lower spread $(\simeq 1 \%)$ is observed for mean ages between 4 and $8 \mathrm{Gyr}$. We therefore
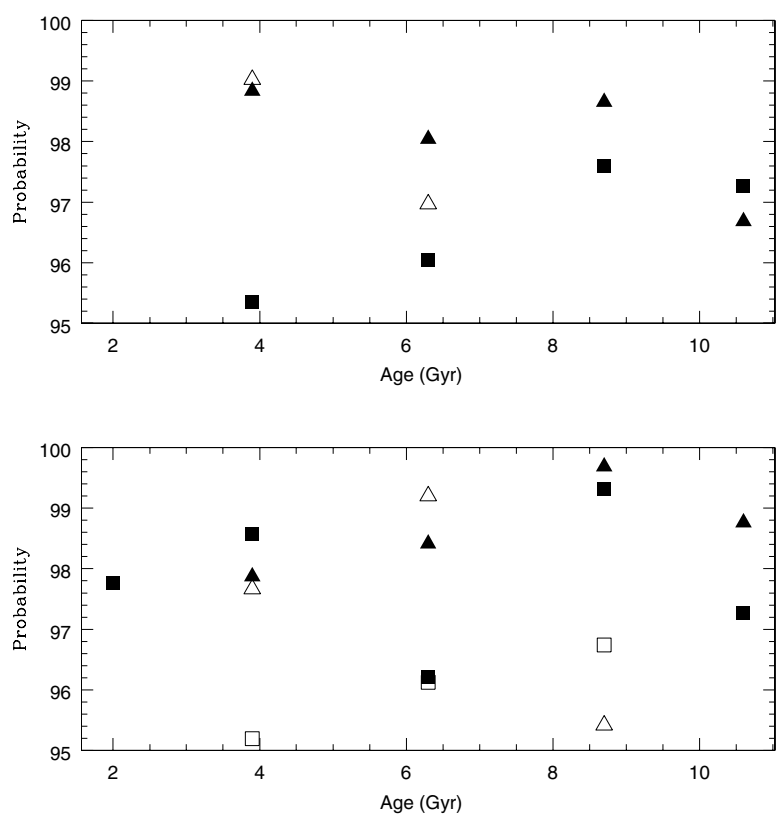

Fig. 10. Probability as a function of mean age for models of a given metallicity which represent the stellar population of SagDIG. Different symbols refer to a different metallicity as follows: $Z=0.0005$ (filled triangles), $Z=0.001$ (empty triangles), $Z=0.004$ (filled squares), $Z=0.008$ (empty squares). Other models explored in this study give a probability much lower than the values plotted here and are not shown. Upper panel: the result obtained considering all the 27 candidate C-stars. Lower panel: the result obtained excluding the three peculiar objects with blue optical colors from the analysis.

conclude that the population of $\mathrm{C}$-stars has to be metal-poor, at least $[\mathrm{Fe} / \mathrm{H}] \lesssim-1.3$, and possibly $[\mathrm{Fe} / \mathrm{H}] \sim-1.6$. The age of the overall population cannot be well constrained, and we can only conclude that the most probable mean age is between 4 and 8 Gyr.

SagDIG is considered one of the most, if not the most metalpoor galaxy in the Local Group. Momany et al. (2005), from the color of its RGB stars, found a metallicity in the range $[\mathrm{Fe} / \mathrm{H}]=$ -2.1 to -1.9 (depending on the adopted reddening) for the oldest stellar population. Their value does not take into account the actual age of RGB stars of SagDIG, so the calculated value is to be considered a lower limit, but the authors assert that the correction to be applied is quite small. On the other hand, from isochrone fitting of young blue loop stars, Momany et al. (2005) found a metallicity $[\mathrm{Fe} / \mathrm{H}] \sim-1.7$ for the $100-600$ Myr old stars. From optical spectroscopy of the brightest H II region Saviane et al. (2002) confirmed that SagDIG is indeed one of the most metal-poor galaxies.

No detailed studies have been dedicated to the SFH of SagDIG so far. Karachentsev et al. (1999) photometry was not deep enough to reach the main sequence turnoff, so they could not resolve the SFH for stars older than 0.2 Gyr. The high precision photometric study of Momany et al. (2005) revealed the presence of a red HB, proving the presence of an old stellar population, with an age comparable with that of Galactic globular clusters. The prominence of the red clump of He burning stars shows that the dominant stellar population is older than $1 \mathrm{Gyr}$. Momany et al. (2005) showed that the distribution in the CMD of red clump and main sequence stars is indicative of an extended period of star formation, spanning from $10 \mathrm{Gyr}$ to $\sim 100 \mathrm{Myr}$ ago. The SFH was not derived in detail by Momany et al. (2005), but 
a forthcoming paper will be dedicated to a further investigation with CMD simulation.

\section{Conclusions}

We investigated the bright AGB content of two Local Group dwarf irregular galaxies DDO 210 and SagDIG using near-IR and optical imaging data.

In DDO 210 we detected two out of three previously known C-stars (Battinelli \& Demers 2000). The third star is located outside the region covered by our data. A further six red objects with $J-K_{\mathrm{s}}>1.1$ and $K_{\mathrm{s}}<18.5$ have optical and near-IR colors consistent with carbon giants. One of these objects, due to its very red color $\left(J-K_{\mathrm{s}}=2.9\right)$, if confirmed with future higher resolution images and/or spectroscopy, could be a dust enshrouded AGB star. With only a handful of C-star candidates it is not possible to put significant limits on the SFH of this galaxy. However, combining these results with much deeper optical imaging may help constrain the rate of star formation over the last few Gyr (for recent results see also McConnachie et al. 2006). Similar, dusty AGB stars have only recently started to be investigated in less distant galaxies thanks to higher sensitivity of near-IR detectors.

SagDIG is a much more luminous galaxy which has experienced a prolonged star formation (Momany et al. 2005), and consequently it harbors a rich intermediate-age population. Due to high foreground contamination, the bluer oxygen-rich AGB stars and bluer C-rich stars are more easily detectable through the narrow-band filter technique (Cook 1987; Demers \& Battinelli 2002). However, the reddest carbon stars and dust enshrouded AGB stars are easier to pick up through near-IR imaging. We have combined the results from the literature with our near-IR images obtained with SOFI at NTT and optical ACS/HST data. All objects redder than the foreground sequence $\left(J-K_{\mathrm{S}}>1.1\right)$ have been carefully inspected and blends or background galaxies were excluded from the final number of $\mathrm{C}$-stars. In the near-IR CMD we identified 27 C-stars candidates in SagDIG. Eighteen of them were C-stars previously known from the works of Demers \& Battinelli (2002) and Cook (1987). Six candidate C-stars identified by Cook (1987) have $K_{\mathrm{s}}$-band magnitudes fainter than the TRGB. Four other candidates by Cook (1987) and one from Demers \& Battinelli (2002) are brighter than the TRGB but cannot be distinguished from foreground stars from our near-IR photometry. Among these 27 candidates, three have peculiar optical colors, too blue compared to the other C-stars. We investigated in some detail their SEDs concluding that they may be either high- $z$ galaxies appearing as point sources in ACS data, or blended stars containing blue and red components.

The LF of the C-stars in near-IR was used to investigate the SFH of SagDIG in the same way as was done for the LMC and SMC (Cioni et al. 2006a,b). The result shows that the underlying stellar population of the galaxy is metal-poor, having at least $[\mathrm{Fe} / \mathrm{H}] \lessgtr-1.3$, and young, with the most probable average age between 4 and 8 Gyr for the dominant population.

Acknowledgements. We thank the anonymous referee for many useful suggestions which improved the presentation. We gratefully acknowledge G. Rodighiero for helpful discussions about the SED, Yazan Momany for his ACS data and useful comments, Alan McConnachie for providing us with the electronic version of his photometry. This publication makes use of data products from the Two Micron All Sky Survey, which is a joint project of the University of Massachusetts and the Infrared Processing and Analysis Center/California Institute of Technology, funded by the National Aeronautics and Space Administration and the National Science Foundation. M.G. wishes to thank the European Southern Observatory for partial funding through DGDF and for hospitality during a visit in which this paper was partially written.

\section{References}

Albert, L., Demers, S., \& Kunkel, W. E. 2000, AJ, 119, 2780

Battinelli, P., \& Demers, S. 2000, AJ, 120, 1801

Battinelli, P., \& Demers, S. 2005, A\&A, 442, 159

Bedin, L. R., Cassisi, S., Castelli, F., et al. 2005, MNRAS, 357, 1038

Bergeat, J., Knapik, A., \& Rutily, B. 2001, A\&A, 369, 178

Bertelli, G., Bressan, A., Chiosi, C., Fagotto, F., \& Nasi, E. 1994, A\&AS, 106, 275

Bruzual, G., \& Charlot, S. 2003, MNRAS, 344, 1000

Carpenter, J. M. 2001, AJ, 121, 2851

Cioni, M.-R. L., \& Habing, H. J. 2005, A\&A, 429, 837

Cioni, M.-R., Loup, C., Habing, H. J., et al. 2000, A\&AS, 144, 235

Cioni, M. R. L., Habing, H. J., Loup, C., Epchtein, N., \& Deul, E. 2004, The Messenger, 115, 22

Cioni, M.-R. L., Girardi, L., Marigo, P., \& Habing, H. J. 2006a, A\&A, 448, 77 Cioni, M.-R. L., Girardi, L., Marigo, P., \& Habing, H. J. 2006b, A\&A, 452, 195 Cohen, M., Wheaton, W. A., \& Megeath, S. T. 2003, AJ, 126, 1090

Cook, K. H. 1987, Ph.D. Thesis

Cutri, R. M., Skrutskie, M. F., van Dyk, S., et al. 2003, 2MASS All Sky Catalog of point sources (The IRSA 2MASS All-Sky Point Source Catalog, NASA/IPAC Infrared Science Archive,

http://irsa.ipac.caltech.edu/applications/Gator/)

Dekker, H., Delabre, B., \& Dodorico, S. 1986, in Instrumentation in astronomy VI; Proc. Meet., Tucson, AZ, Mar. 4-8, 1986, Part 1 (A87-36376 1535), Bellingham, WA, Society of Photo-Optical Instrumentation Engineers, ed. D. L. Crawford, 339

Demers, S., \& Battinelli, P. 2002, AJ, 123, 238

Demers, S., Battinelli, P., \& Artigau, E. 2006, A\&A, 456, 905

Girardi, L., Bressan, A., Bertelli, G., \& Chiosi, C. 2000, A\&AS, 141, 371

Girardi, L., Bertelli, G., Chiosi, C., \& Marigo, P. 2003, in IAU Symp., ed. K. van der Hucht, A. Herrero, \& C. Esteban, 551

Girardi, L., Groenewegen, M. A. T., Hatziminaoglou, E., \& da Costa, L. 2005, A\&A, 436, 895

Groenewegen, M. A. T. 2004, A\&A, 425, 595

Gullieuszik, M., Held, E. V., Momany, Y., et al. 2005, in Near-fields cosmology with dwarf elliptical galaxies, ed. H. Jerjen, \& B. Binggeli, IAU Colloq., 198, 47

Gullieuszik, M., Held, E. V., Rizzi, L., et al. 2007, A\&A, 467, 1025

Kang, A., Sohn, Y.-J., Rhee, J., et al. 2005, A\&A, 437, 61

Karachentsev, I., Aparicio, A., \& Makarova, L. 1999, A\&A, 352, 363

Lançon, A., \& Mouhcine, M. 2002, A\&A, 393, 167

Lee, M. G., Aparicio, A., Tikonov, N., Byun, Y.-I., \& Kim, E. 1999, AJ, 118, 853

Lo, K. Y., Sargent, W. L. W., \& Young, K. 1993, AJ, 106, 507

Maraston, C., Daddi, E., Renzini, A., et al. 2006, ApJ, 652, 85

Marigo, P., Girardi, L., \& Bressan, A. 1999, A\&A, 344, 123

Marigo, P., Girardi, L., \& Chiosi, C. 2003, A\&A, 403, 225

McConnachie, A. W., Arimoto, N., Irwin, M., \& Tolstoy, E. 2006, MNRAS, 373, 715

Momany, Y., Held, E. V., Saviane, I., \& Rizzi, L. 2002, A\&A, 384, 393

Momany, Y., Held, E. V., Saviane, I., et al. 2005, A\&A, 439, 111

Moorwood, A., Cuby, J.-G., \& Lidman, C. 1998, The Messenger, 91,9

Nikolaev, S., \& Weinberg, M. D. 2000, ApJ, 542, 804

Nowotny, W., Kerschbaum, F., Schwarz, H. E., \& Olofsson, H. 2001, A\&A, 367, 557

Persson, S. E., Murphy, D. C., Krzeminski, W., Roth, M., \& Rieke, M. J. 1998, AJ, 116, 2475

Raimondo, G., Cioni, M.-R. L., Rejkuba, M., \& Silva, D. R. 2005, A\&A, 438, 521

Rejkuba, M., Minniti, D., Silva, D. R., \& Bedding, T. R. 2001, A\&A, 379, 781

Rejkuba, M., Greggio, L., Harris, W. E., Harris, G. L. H., \& Peng, E. W. 2005, ApJ, 631, 262

Renzini, A., \& Buzzoni, A. 1986, in Spectral Evolution of Galaxies, ed. C. Chiosi, \& A. Renzini, ASSL, 122, 195

Rieke, G. H., \& Lebofsky, M. J. 1985, ApJ, 288, 618

Salaris, M., Chieffi, A., \& Straniero, O. 1993, ApJ, 414, 580

Saviane, I., Rizzi, L., Held, E. V., Bresolin, F., \& Momany, Y. 2002, A\&A, 390, 59

Stetson, P. B. 1987, PASP, 99, 191

Stetson, P. B. 1993, in Stellar Photometry - Current Techniques and Future Developments, ed. C. J. Butler, \& I. Elliott, IAU Colloq., 136, 291

Stetson, P. B. 1994, PASP, 106, 250

Stetson, P. B. 2000, PASP, 112, 925

Totten, E. J., Irwin, M. J., \& Whitelock, P. A. 2000, MNRAS, 314, 630

Valenti, E., Ferraro, F. R., \& Origlia, L. 2004, MNRAS, 354, 815 


\section{Online Material}




\section{Appendix A: The SED of the three peculiar objects}

We point out the strange colors of the three red stars (number 6, 7 and 12) with near-IR colors typical of C-stars but not classified as C-stars by previous studies. In the $\left(J-K_{\mathrm{s}}\right)$ vs. $\left(V-K_{\mathrm{s}}\right)$ diagram of Fig. 7 they are the only stars with $V-K_{\mathrm{s}}<3.5$ (the other star is the blue object classified as blended, see Fig. 6). In the $\left(J-K_{\mathrm{s}}\right)$ vs. $\left(I-K_{\mathrm{s}}\right)$ diagram of Fig. 8 only two of them have blue optical colors, bluer than $I-K_{\mathrm{s}}=2$. The third one (number 6) is placed among "normal" C-stars, at $I-K_{\mathrm{s}}=2.24$. We can therefore say that these three stars show SEDs which are similar to a C-star SED in the near-IR, but have a blue excess, which is less pronounced for star number 6 . We explore the following possible explanations: i) they are high- $z$ galaxies with a distance sufficient to make them appear as point-like sources and producing a SED that could explain their color; ii) they are double stars composed of a very red and a very blue star. The latter hypothesis could be due to a blend of two stars or an intrinsic binary.

We derived the SEDs of these three objects using the zero point taken from Bedin et al. (2005) for the ACS photometry and from Cohen et al. (2003) for the near-IR. First we tested the high redshift galaxy hypothesis, reproducing the observed SEDs with templates generated with the stellar population synthesis code GALAXEV (Bruzual \& Charlot 2003). Our best fit models are shown in the upper panels of Fig. A.1. The SEDs are compatible with a young $\sim 10^{10} M_{\odot}$ galaxy at redshift $z \simeq 2.5$ for objects number 7 and 12 , and $z \simeq 0.5$ for object number 6 . In a standard cosmological model $\left(H_{0}=71, \Omega_{\mathrm{M}}=0.3, \Omega_{\Lambda}=0.7\right) z=2.5$ corresponds to a luminosity distance $D_{L} \simeq 20000 \mathrm{Mpc}$. The ACS scale is 0 . $^{\prime} 05$ pixel $^{-1}$, hence a pixel corresponds to $\sim 5 \mathrm{kpc}$ for an object located at $20000 \mathrm{Mpc}$. Given that the typical PSF is 4 pixels, a point-like source located at $z=2.5$ must have a $F W H M<20 \mathrm{kpc}$. This is reasonable for a $10^{10} M_{\odot}$ galaxy in which we actually observe only the youngest stars forming possibly in the central regions observed in the rest-frame ultra-violet with ACS. We therefore conclude that two objects (number 7 and 12) could be galaxies at $z \simeq 2.5$. The third one, at $z \simeq 0.5$, should be located at a distance of an order of magnitude smaller and thus should be one order of magnitude smaller, in order to appear as a point like source. Such a small distance is incompatible with a $10^{10} M_{\odot}$ galaxy. The purpose of the discussion is to show the viability of the galaxy hypothesis, and hence a detailed discussion of other possible solutions within the range of explored parameters is beyond the scope of this paper.

Another possible explanation is that they are double stars or a pair of unresolved stars, composed of a red and a blue star. The resultant sum of their SEDs would have blue optical colors and red near-IR colors. To test this hypothesis, we used the spectral library of C-stars from Lançon \& Mouhcine (2002). We added to a C-star the SED of a simple black body. We consider this a good approximation of a moderately hot star, sufficient for the qualitative aim of this analysis. In the central panels of Fig. A.1 the resulting fits are presented and appear to be reasonably good. We derived the magnitudes of the two components of the best fit models. In the lower panels of Fig. A.1 the optical magnitudes of the C-star and the black body are shown in the ACS CMD of SagDIG.

All three objects are compatible with a SED composed of a carbon star and a very young main sequence star. However, we note that the optical magnitudes of the carbon star components place them at distances that are not compatible with SagDIG distance moduli. The red star component could be a foreground star belonging to the galactic population and therefore not necessarily carbon giant, but also a carbon or M-dwarf.

Therefore we conclude that the solution with the two star SED points towards the presence of a blend rather than a binary star. For two objects there is also a possibility that they are high redshift $(z \sim 2.5)$ galaxies. A conclusive test would need a spectroscopic analysis of the three objects, which is clearly beyond the scope of the present paper. 
M. Gullieuszik et al.: Near-infrared photometry of the Sagittarius dwarf irregular galaxy and DDO 210, Online Material p 3

Object $n^{\circ} 6$

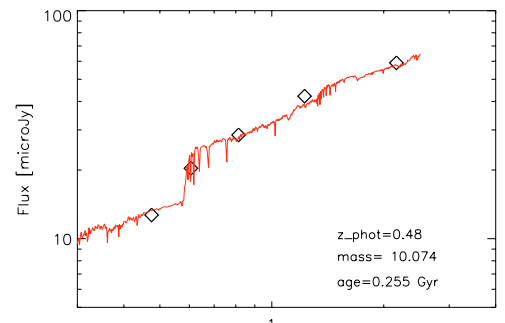

wavelength [micron]
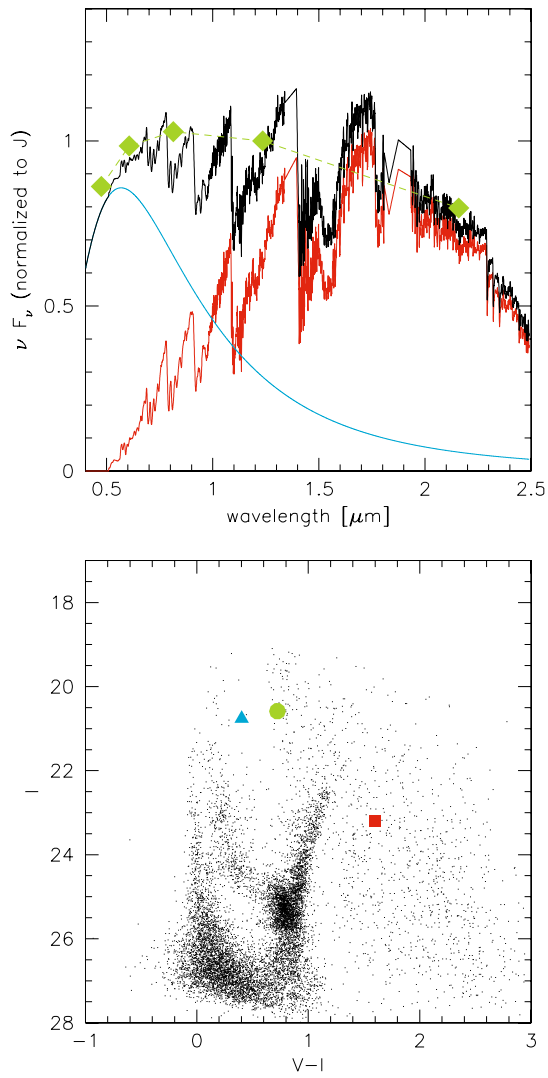

Object $n^{\circ} 7$

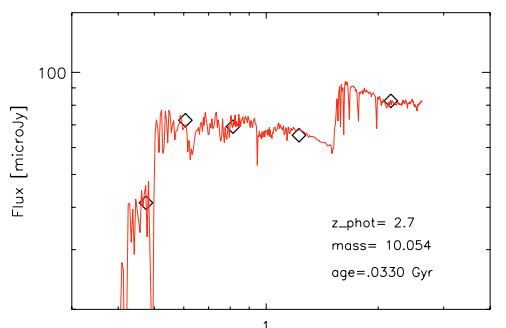

wovelength [micron]
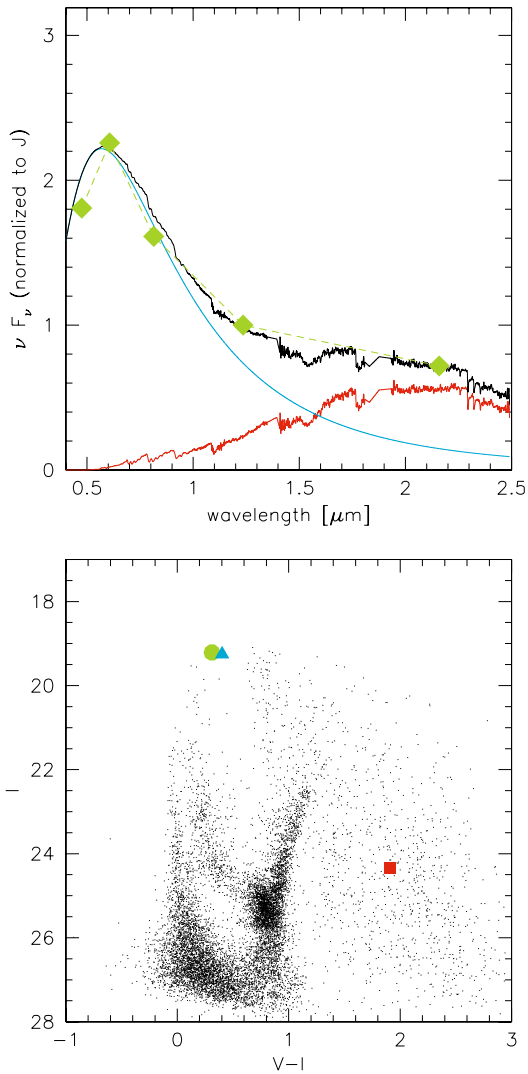

Object $n^{\circ} 12$
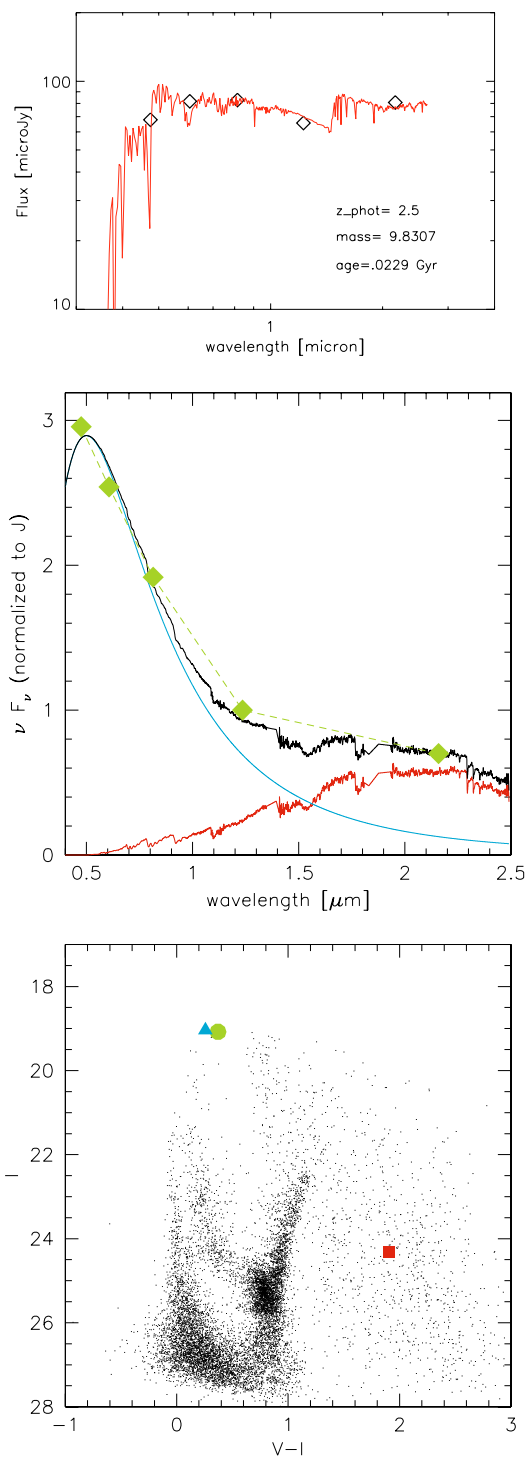

Fig. A.1. The SED of objects number 6, 7 and 12. Upper panels: the flux together with the best fit models of distant galaxies. Central panels: the flux density together with the double star models: the SED is fitted by the sum of a carbon star spectrum taken from Lançon \& Mouhcine (2002) and a black body. Lower panels: the ACS CMD of SagDIG and the photometric points calculated from the black body model (triangles) and the carbon star spectra (squares). The measured magnitudes of the real objects are plotted as circles. 